Louisiana State University

LSU Digital Commons

Faculty Publications

Department of Mathematics

$5-1-2008$

\title{
A characterization of box-mengerian matroid ports
}

Xujin Chen

Institute of Applied Mathematics, AMSS, CAS

Guoli Ding

Louisiana State University

Wenan Zang

The University of Hong Kong

Follow this and additional works at: https://digitalcommons.Isu.edu/mathematics_pubs

\section{Recommended Citation}

Chen, X., Ding, G., \& Zang, W. (2008). A characterization of box-mengerian matroid ports. Mathematics of Operations Research, 33 (2), 497-512. https://doi.org/10.1287/moor.1070.0306

This Article is brought to you for free and open access by the Department of Mathematics at LSU Digital Commons. It has been accepted for inclusion in Faculty Publications by an authorized administrator of LSU Digital Commons. For more information, please contact ir@lsu.edu. 


\title{
A Characterization of Box-Mengerian Matroid Ports
}

\author{
Xujin Chen ${ }^{1}$ \\ Institute of Applied Mathematics, Chinese Academy of Sciences, Beijing, China \\ Guoli Ding ${ }^{2}$ \\ Mathematics Department, Louisiana State University, Baton Rouge, Louisiana, USA \\ Wenan Zang ${ }^{3}$ \\ Department of Mathematics, The University of Hong Kong, Hong Kong, China
}

\begin{abstract}
Let $M$ be a matroid on $E \cup\{\ell\}$, where $\ell \notin E$ is a distinguished element of $M$. The $\ell$-port of $M$ is the set $\mathcal{P}=\{P: P \subseteq E$ with $P \cup\{\ell\}$ a circuit of $M\}$. Let $A$ be the $\mathcal{P}$ - $E$ incidence matrix. Let $U_{2,4}$ be the uniform matroid on four elements of rank two, $F_{7}$ be the Fano matroid, $F_{7}^{*}$ be the dual of $F_{7}$, and $F_{7}^{+}$be the unique series extension of $F_{7}$. In this paper, we prove that the system $A \boldsymbol{x} \geq \mathbf{1}, \boldsymbol{x} \geq \mathbf{0}$ is box-totally dual integral (box-TDI) if and only if $M$ has no $U_{2,4}$-minor using $\ell$, no $F_{7}^{*}$-minor using $\ell$, and no $F_{7}^{+}$-minor using $\ell$ as a series element. Our characterization yields a number of interesting results in combinatorial optimization.
\end{abstract}

MSC 2000 subject classification. Primary: 90C10, 90C27, 90C57.

OR/MS subject classification. Primary: Programming/graphs.

Key words. Binary clutter, Binary Matroid, Regular Matroid, Box-Total Dual Integrality.

\footnotetext{
${ }^{1}$ Supported in part by NSF of China under Grant No. 10771209. E-mail: xchen@amss.ac.cn.

${ }^{2}$ Supported in part by NSA grant H98230-05-1-0081 and NSF grant DMS-0556091. E-mail: ding@math.lsu.edu.

${ }^{3}$ Supported in part by the Research Grants Council of Hong Kong. E-mail: wzang@maths.hku.hk.
} 


\section{Introduction}

A linear system $A \boldsymbol{x} \geq \boldsymbol{b}, \boldsymbol{x} \geq \mathbf{0}$ is called totally dual integral (TDI) if the maximum in the LP-duality equation

$$
\min \left\{\boldsymbol{w}^{T} \boldsymbol{x}: A \boldsymbol{x} \geq \boldsymbol{b}, \boldsymbol{x} \geq \mathbf{0}\right\}=\max \left\{\boldsymbol{y}^{T} \boldsymbol{b}: \boldsymbol{y}^{T} A \leq \boldsymbol{w}^{T}, \boldsymbol{y} \geq \mathbf{0}\right\}
$$

has an integral optimal solution $\boldsymbol{y}$, for every integral vector $\boldsymbol{w}$ for which the maximum is finite. As shown by Edmonds and Giles [7], if $A \boldsymbol{x} \geq \boldsymbol{b}, \boldsymbol{x} \geq \mathbf{0}$ is a TDI system and $\boldsymbol{b}$ is integral, then the minimum also has an integral optimal solution provided the optimum of (1.1) is finite. So total dual integrality has been serving as a general framework for the study of min-max relations in combinatorial optimization. In various problem settings, $A \boldsymbol{x} \geq \boldsymbol{b}, \boldsymbol{x} \geq \mathbf{0}$ can be shown to have the stronger property that, for every choice of rational vectors $\boldsymbol{u}$ and $\boldsymbol{l}$, the system $A \boldsymbol{x} \geq \boldsymbol{b}, \boldsymbol{x} \geq \mathbf{0}, \boldsymbol{u} \geq \boldsymbol{x} \geq \boldsymbol{l}$ is TDI; such a linear system $A \boldsymbol{x} \geq \boldsymbol{b}, \boldsymbol{x} \geq \mathbf{0}$ is called box-totally dual integral (box-TDI). For detailed information about box-TDI systems, see Cook [3] and Edmonds and Giles [8].

In this paper a box-TDI system associated with matroids is to be established. We introduce some notions before presenting our result. A 0-1 matrix $A$ is called Mengerian [18] if the system $A \boldsymbol{x} \geq \mathbf{1}, \boldsymbol{x} \geq \mathbf{0}$ is TDI; the matrix is so named because for path-edge incidence matrices $A$, (meaning that there exists a graph $G$, together with two distinct vertices $s$ and $t$, such that rows of $A$ are precisely the incidence vectors of the edge sets of all st-paths), the statement " $A$ is Mengerian" is exactly the edge version of Menger's Theorem. Naturally, $A$ is box-Mengerian if the system $A \boldsymbol{x} \geq \mathbf{1}, \boldsymbol{x} \geq \mathbf{0}$ is box-TDI.

A clutter [6] on a finite set $E$ is a set $\mathcal{L}$ of subsets of $E$ such that no member of $\mathcal{L}$ is a proper subset of another member. We shall call $\mathcal{L}$ Mengerian (resp. box-Mengerian) if $\mathcal{L}=\{\emptyset\}, \mathcal{L}=\emptyset$, or the $\mathcal{L}$ - $E$ incidence matrix $A_{\mathcal{L}}$ is Mengerian (resp. box-Mengerian). Notice that clutters $\mathcal{L}$ and 0-1 matrices $A_{\mathcal{L}}$ are essentially the same class of objects. We shall use the language of clutters since it is more combinatorial.

As usual, let $U_{2,4}$ be the uniform matroid on four elements of rank two, let $F_{7}$ be the Fano matroid, let $F_{7}^{*}$ be the dual of $F_{7}$, and let $F_{7}^{+}$be the unique series extension of $F_{7}$. We refer to Oxley [13] for an in-depth account of matroid theory and undefined terms.

Let $M$ be a matroid [13] on $E \cup\{\ell\}$, where $\ell \notin E$ is a distinguished element of $M$. A matroid obtained from $M$ by deleting and contracting elements in $E$ is called a minor of $M$ using $\ell$. The $\ell$-port of $M$ [12] is the clutter $\mathcal{P}_{M, \ell}=\{P: P \subseteq E$ with $P \cup\{\ell\}$ a circuit of $M\}$ on $E$. In [18], Seymour characterized all pairs $(M, \ell)$ for which $\mathcal{P}_{M, \ell}$ is Mengerian.

Theorem 1.1 (Seymour [18]) Let $M$ be a matroid and let $\ell$ be an element of $M$. Then $\mathcal{P}_{M, \ell}$ is Mengerian if and only if $M$ has neither $U_{2,4}$-minor using $\ell$ nor $F_{7}^{*}$-minor using $\ell$.

This far-reaching theorem, which yields a number of important min-max relations in combinatorial optimization, has attracted tremendous research efforts in matroid optimization, see, for instances, Gerards and Laurent [9], Guenin [10], Truemper [20], Tseng and Truemper [21], and Ding and Zang [5]. For any matrix $A$ and any vectors $\boldsymbol{l}, \boldsymbol{u}$, let $Q(A, \boldsymbol{l}, \boldsymbol{u})$ denote the polytope $\{\boldsymbol{x}: A \boldsymbol{x} \geq \mathbf{1}, \boldsymbol{x} \geq \mathbf{0}\} \cap\{\boldsymbol{x}: \boldsymbol{l} \leq \boldsymbol{x} \leq \boldsymbol{u}\}$. A vector $\boldsymbol{x}$ is called $\frac{1}{d}$-integral, where $d$ is a positive integer, if all coordinates of $d \boldsymbol{x}$ are integral. Gerards and Laurent obtained the following structural theorem, which can be found in several interesting applications $[9,11]$.

Theorem 1.2 (Gerards and Laurent [9]) Let $M$ be a matroid on $E \cup\{\ell\}$ with $\ell \notin E$, and let $A$ be the $\mathcal{P}_{M, \ell}-E$ incidence matrix. Then all vertices of $Q(A, \boldsymbol{l}, \boldsymbol{u})$ are $\frac{1}{d}$-integral, for all positive integers $d$ and all $\frac{1}{d}$-integral vectors $\boldsymbol{l}, \boldsymbol{u}$, if and only if $M$ has no $U_{2,4}$-minor using $\ell$, no $F_{7}^{*}$-minor using $\ell$, and no $F_{7}^{+}$-minor using $\ell$ as a series element. 
The purpose of this paper is to present a characterization of all pairs $(M, \ell)$ for which $\mathcal{P}_{M, \ell}$ is boxMengerian.

Theorem 1.3 Let $M$ be a matroid and let $\ell$ be an element of $M$. Then $\mathcal{P}_{M, \ell}$ is box-Mengerian if and only if $M$ has no $U_{2,4}$-minor using $\ell$, no $F_{7}^{*}$-minor using $\ell$, and no $F_{7}^{+}$-minor using $\ell$ as a series element.

Let $A$ be as defined in Theorem 1.2. Our theorem asserts that $M$ contains no aforementioned minors if and only if, for any rational vectors $\boldsymbol{l}$ and $\boldsymbol{u}$, the maximum of the following LP-duality equation

$$
\min \left\{\boldsymbol{w}^{T} \boldsymbol{x}: A \boldsymbol{x} \geq \mathbf{1}, \boldsymbol{x} \geq \mathbf{0}, \boldsymbol{u} \geq \boldsymbol{x} \geq \boldsymbol{l}\right\}=\max \left\{\boldsymbol{\alpha}^{T} \mathbf{1}+\boldsymbol{\beta}^{T} \boldsymbol{l}-\boldsymbol{\gamma}^{T} \boldsymbol{u}: \boldsymbol{\alpha}^{T} A+\boldsymbol{\beta}^{T}-\boldsymbol{\gamma}^{T} \leq \boldsymbol{w}^{T}, \boldsymbol{\alpha}, \boldsymbol{\beta}, \boldsymbol{\gamma} \geq \mathbf{0}\right\}
$$

has an integral optimal solution, for any integral vector $\boldsymbol{w}$ for which the optimum is finite, and so does the minimum provided both $\boldsymbol{l}$ and $\boldsymbol{u}$ are integral.

Observe that the excluded minors of the above two theorems are exactly the same. We shall prove in Section 7 that, if a 0-1 matrix $A$ is box-Mengerian then for all positive integers $d$ and all $\frac{1}{d}$-integral vectors $\boldsymbol{l}$ and $\boldsymbol{u}$, all vertices of $Q(A, \boldsymbol{l}, \boldsymbol{u})$ are $\frac{1}{d}$-integral. Therefore, Theorem 1.3 implies Theorem 1.2; yet, box $\frac{1}{d}$-integrality for all $d$ does not imply box-total dual integrality in general. So the integrality property for the maximum in the above equation cannot be deduced directly from Theorem 1.2. In Section 7, we shall also discuss applications of our main result to $T$-joins, $T$-cuts, two-commodity paths, two-commodity cuts, and odd cycles in graphs. We shall see that Theorem 1.3 strengthens Theorem 1.1 in many cases. Another motivation of our work is the following. Although there are many nice results on total dual integrality, things become much more difficult when the stronger notion of box-total dual integrality is considered. Few proof techniques have been found that are applicable to this stronger property [15]. To our knowledge, most of the known box-TDI systems $A \boldsymbol{x} \leq \boldsymbol{b}$ can be established by checking that $A$ is totally unimodular or that for any rational $\boldsymbol{c}$, the problem $\max \left\{\boldsymbol{c}^{T} \boldsymbol{x}: A \boldsymbol{x} \leq \boldsymbol{b}\right\}$ has (if finite) an optimal solution $\boldsymbol{y}$ such that the rows of $A$ corresponding to positive components of $\boldsymbol{y}$ form a totally unimodular submatrix of $A$ (see Theorem 5.35 of Schrijver [15]). However, the box-TDI system considered in this paper, which is fairly "large", can only be derived in a sophisticated way; our proof of Theorem 1.3 might suggest more insights into box-total dual integrality.

Our main result can also be stated in terms of clutters. Let $\mathcal{L}$ be a clutter on $E$. We define deletion and contraction operations on $\mathcal{L}$ as follows. For any $e \in E$, let $\mathcal{L} \backslash e$ consist of members $X$ of $\mathcal{L}$ with $e \notin X$ and let $\mathcal{L} / e$ consist of minimal members of $\{X-\{e\}: X \in \mathcal{L}\}$. Clearly, both $\mathcal{L} \backslash e$ and $\mathcal{L} / e$ are clutters on $E-\{e\}$. A clutter obtained from $\mathcal{L}$ by applying a sequence of deletions and contractions is called a minor [16] of $\mathcal{L}$. If $\mathcal{L}$ is a port of a matroid $M$, it is easy to verify that taking minors in $M$ and taking minors in $\mathcal{L}$ are the same thing, as pointed out in [18].

Proposition 1.1 Let $\ell$ be an element of matroid $M$ and let $\mathcal{L}$ be the $\ell$-port of $M$. For any element $e \neq \ell$ of $M$, the $\ell$-port of $M \backslash e$ is $\mathcal{L} \backslash$ e and the $\ell$-port of $M / e$ is $\mathcal{L} / e$.

We also need to consider three special ports. By symmetry, all ports of $U_{2,4}$ are isomorphic and so are all ports of $F_{7}^{*}$. We denote these two clutters by $C_{3}$ and $Q_{6}$, respectively. Similarly, if $\ell_{1}$ and $\ell_{2}$ are the two element of $F_{7}^{+}$that are in series, then the $\ell_{1}$-port and $\ell_{2}$ port of $F_{7}^{+}$are isomorphic, which we denote by $Q_{7}$. In other words, $C_{3}$ is the clutter on $\{1,2,3\}$ that consists of sets $\{1,2\},\{2,3\}$, and $\{1,3\} ; Q_{6}$ is the clutter on $\{1,2,3,4,5,6\}$ that consists of sets $\{1,3,5\},\{1,2,6\},\{2,3,4\}$, and $\{4,5,6\}$; and $Q_{7}$ is the clutter on $\{1,2,3,4,5,6,7\}$ that consists of sets $\{1,4,7\},\{2,5,7\},\{3,6,7\},\{1,2,6,7\},\{1,3,5,7\},\{2,3,4,7\}$, and $\{4,5,6,7\}$. Clearly, Proposition 1.1 implies that Theorem 1.3 can be restated as follows. 
Corollary 1.1 A port is box-Mengerian if and only if none of $C_{3}, Q_{6}$, and $Q_{7}$ is its minor.

Ports with no $C_{3}$-minors are known as binary clutters, which are precisely ports of binary matroids [16]. Corollary 1.1 clearly implies the following, which is basically equivalent to our main result.

Corollary 1.2 A binary clutter is box-Mengerian if and only if neither $Q_{6}$ nor $Q_{7}$ is its minor.

We now outline the rest of the paper. The proof of the necessity part of Theorem 1.3 is straightforward, which we give in Section 2. The proof of the sufficiency part is much more difficult. Let $\Im$ be the set of pairs $(M, \ell)$, where $M$ is a connected matroid on at least two elements, including $\ell$, such that $M$ has no $U_{2,4}$-minor using $\ell$, no $F_{7}^{*}$-minor using $\ell$, and no $F_{7}^{+}$-minor using $\ell$ as a series element. We first discuss in Section 3 results of Tseng and Truemper [21] and Gerards and Laurent [9], which say that, for every $(M, \ell)$ in $\Im$, either $M$ is regular or $M / \ell$ is not 3-connected. This result allows us to decompose matroids in $\Im$ into regular matroids. In Section 4 we prove that $\mathcal{P}_{M, \ell}$ is box-Mengerian if $M$ is regular. In Section 5 we analyze feasible solutions to the LP problem that is in our consideration. In particular, we study the behavior of such a solution when the corresponding matroids are composed or decomposed. We complete our proof of Theorem 1.3 in Section 6. Finally, in the last section, we discuss several applications of our main result.

We close this section by defining some notations that we shall use in this paper. Let $\mathbb{Q}$ and $\mathbb{Z}$ be the sets of rationals and integrals, respectively. Let $\mathbb{Q}_{+}$and $\mathbb{Z}_{+}$be the sets of nonnegative numbers in the corresponding sets. For any two sets $\Omega$ and $K$, where $\Omega$ is always a set of numbers and $K$ is always finite, we use $\Omega^{K}$ to denote the set of vectors $\boldsymbol{x}=(\boldsymbol{x}(k): k \in K)$ whose coordinates are members of $\Omega$. Suppose $J \subseteq K$. The $|J|$-dimensional vector $\left.\boldsymbol{x}\right|_{J}=(\boldsymbol{x}(j): j \in J)$ stands for the projection of $\boldsymbol{x}$ to $\Omega^{J}$. In addition, $\boldsymbol{x}(J)$ denotes the value $\sum_{j \in J} \boldsymbol{x}(j)$. The support of $\boldsymbol{x}$, denoted by spt(x), is the set $\{k \in K: \boldsymbol{x}(k) \neq 0\}$. Finally, we use $\overline{\boldsymbol{x}}$ to denote the vector $(|\boldsymbol{x}(k)|: k \in K)$.

\section{Necessity}

In this section we prove the "only if" part of Theorem 1.3. We begin with a lemma (see Theorem 22.7 in [14]), which says that in the definition of box-TDI systems, we may allow coordinates of $\boldsymbol{u}$ and $\boldsymbol{l}$ to be $\infty$.

Lemma 2.1 If $A \boldsymbol{x} \geq \boldsymbol{b}, x \geq \mathbf{0}$ is box-TDI, then $A \boldsymbol{x} \geq \boldsymbol{b}, x \geq \mathbf{0}, \boldsymbol{u} \geq \boldsymbol{x} \geq \boldsymbol{l}$ is TDI, for any $\boldsymbol{u}$ with coordinates in $\mathbb{Q} \cup\{+\infty\}$ and any $\boldsymbol{l}$ with coordinates in $\mathbb{Q} \cup\{-\infty\}$. In particular, $A \boldsymbol{x} \geq \boldsymbol{b}, \boldsymbol{x} \geq \mathbf{0}$ is TDI.

Proof of the necessity part of Theorem 1.3. Let $M$ be a matroid on $E \cup\{\ell\}$, where $\ell \notin E$, such that $\mathcal{P}_{M, \ell}$ is box-Mengerian. It was proved in lemmas 2.2-2.4 of [2] that minors of a box-Mengerian clutter remains box-Mengerian. Therefore, if $M^{\prime}$ is a minor of $M$ using $\ell$, we deduce from Proposition 1.1 that $\mathcal{P}_{M^{\prime}, \ell}$ is also box-Mengerian. It remains to show that $C_{3}, Q_{6}$, and $Q_{7}$ are not box-Mengerian. A straightforward computation shows that $C_{3}$ and $Q_{6}$ are not Mengerian [18]. Thus Lemma 2.1 implies that they are not box-Mengerian either. Next, let $A=A_{Q_{7}}$ be the incidence matrix of $Q_{7}$. We choose $\boldsymbol{w}=(1,1,1,1,1,1,2)^{T}$, $\boldsymbol{l}=\left(0, \frac{1}{2}, 0, \frac{1}{2}, 0, \frac{1}{2}, 0\right)^{T}$, and $\boldsymbol{u}=\left(\frac{1}{2}, \frac{1}{2}, \frac{1}{2}, \frac{1}{2}, \frac{1}{2}, \frac{1}{2}, \frac{1}{2}\right)^{T}$. It is routine to verify that $\boldsymbol{x}=\left(\frac{1}{4}, \frac{1}{2}, \frac{1}{4}, \frac{1}{2}, \frac{1}{4}, \frac{1}{2}, \frac{1}{4}\right)^{T}$ and $\boldsymbol{y}=\left(\frac{1}{2}, \frac{1}{2}, \frac{1}{2}, 0, \frac{1}{2}, 0,0,0, \frac{1}{2}, 0, \frac{1}{2}, 0, \frac{1}{2}, 0,0,0,0,0,0,0,0\right)^{T}$ are feasible solutions to the pair of LP problems

$$
\min \left\{\boldsymbol{w}^{T} \boldsymbol{x} \mid\left[\begin{array}{c}
A \\
I \\
-I
\end{array}\right] \boldsymbol{x} \geq\left[\begin{array}{c}
\mathbf{1} \\
\boldsymbol{l} \\
-\boldsymbol{u}
\end{array}\right], \boldsymbol{x} \geq \mathbf{0}\right\} \quad \text { and } \quad \max \left\{\boldsymbol{y}^{T}\left[\begin{array}{c}
\mathbf{1} \\
\boldsymbol{l} \\
-\boldsymbol{u}
\end{array}\right] \mid \boldsymbol{y}^{T}\left[\begin{array}{c}
A \\
I \\
-I
\end{array}\right] \leq \boldsymbol{w}^{T}, \boldsymbol{y} \geq \mathbf{0}\right\} .
$$


Moreover, $\boldsymbol{x}$ and $\boldsymbol{y}$ achieve the same objective value $\frac{11}{4}$, which implies that this is the optimal value for both problems. Since $\left(\mathbf{1}^{T}, \boldsymbol{l}^{T},-\boldsymbol{u}^{T}\right)$ is $\frac{1}{2}$-integral yet $\frac{11}{4}$ is not, we conclude that no optimal solution to the maximization problem is integral. Therefore, by definition, $\mathcal{P}_{F_{7}^{+}, \ell}=Q_{7}$ is not box-Mengerian.

\section{Matroid decomposition}

The goal of this section is to introduce results on matroids that will be used in proving our main result.

\subsection{Connectivity and summing operations}

We begin with the definition of matroid connectivity. Let $M$ be a matroid on $E$. A partition $\left(E_{1}, E_{2}\right)$ of $E$ is called a $k$-separation, where $k$ is a positive integer, if $\min \left\{\left|E_{1}\right|,\left|E_{2}\right|\right\} \geq k$ and $r\left(E_{1}\right)+r\left(E_{2}\right) \leq r(E)+k-1$. A matroid is $k$-connected if it has no $k^{\prime}$-separation, for any $k^{\prime}<k$. As customary, 2-connected matroids are called connected and others are disconnected.

For $i=1,2$, let $M_{i}$ be a matroid on $E_{i}$ with $E_{1} \cap E_{2}=\emptyset$. The 1-sum of $M_{1}$ and $M_{2}$ is the matroid $M$ on $E_{1} \cup E_{2}$ such that $C$ is a circuit of $M$ if and only if it is a circuit of either $M_{1}$ or $M_{2}$. Next, for $i=1,2$, let $M_{i}$ be a matroid on $E_{i} \cup\{p\}$, where $E_{1} \cap E_{2}=\emptyset$ and $p \notin E_{1} \cup E_{2}$. The parallel connection of $M_{1}$ and $M_{2}$ is the matroid $M$ on $E_{1} \cup E_{2} \cup\{p\}$ such that $C$ is a circuit of $M$ if and only if $C$ is a circuit of $M_{1}$ or $M_{2}$, or $C=\left(C_{1}-\{p\}\right) \cup\left(C_{2}-\{p\}\right)$, where, for $i=1,2, C_{i}$ is a circuit of $M_{i}$ that contains $p$. Matroid $M \backslash p$ is called the 2-sum of $M_{1}$ and $M_{2}$. The following are well known results, which can be found in [13] (see 4.2.13, 8.3.1, 7.1.20, and 7.1.19).

Lemma 3.1 (i) If a matroid $M$ is disconnected then it is the 1-sum of two nonempty matroids.

(ii) If a connected matroid $M$ has a 2-separation $\left(E_{1}, E_{2}\right)$, then $M$ is the 2-sum of connected matroids $M_{1}$ and $M_{2}$ such that $E\left(M_{i}\right)=E_{i} \cup\{p\}(i=1,2)$, where $p \notin E$. In addition, for $i=1,2$, there exist disjoint subsets $X_{i}$ and $Y_{i}$ of $E-E_{i}$ such that $M_{i} \cong M \backslash X_{i} / Y_{i}$.

We shall refer to $M_{1}$ and $M_{2}$ determined in (ii) as matroids induced by $\left(E_{1}, E_{2}\right)$. Their common element will always be denoted by $p$.

\subsection{Matroids in $\Im$}

In this subsection we describe the structure of matroids in $\Im$. This structure is the foundation for our proof. We begin with a well known result of Bixby [1], which implies that all matroids in $\Im$ are binary.

Theorem 3.1 Let $\ell$ be an element of a connected matroid $M$. Then $M$ has no $U_{2,4}$-minor using $\ell$ if and only if $M$ is binary.

Seymour proved several similar results in [17], including the following.

Theorem 3.2 Let $\ell$ be an element of a connected matroid $M$. Then $M$ has no $U_{2,4}$-minor using $\ell$, no $F_{7}$-minor using $\ell$, and no $F_{7}^{*}$-minor using $\ell$ if and only if $M$ is regular.

The next is a result of the same type, due to Tseng and Truemper [21], which deals with $F_{7}^{*}$. Our formulation is slightly weaker than the original result, see [9]. 
Theorem 3.3 Let $\ell$ be an element of a binary matroid $M$. If $M$ has no $F_{7}^{*}$-minor using $\ell$, then at least one of the following holds:

(i) $M$ is isomorphic to $F_{7}$;

(ii) $M$ is regular;

(iii) $M$ is not 3-connected;

(iv) $M$ is 3-connected but $M / \ell$ is not.

Based on this result, Gerards and Laurent [9] obtained a structural result for matroids in $\Im$.

Theorem 3.4 If $(M, \ell) \in \Im$ then either $M$ is regular or $M / \ell$ is not 3-connected.

\subsection{Signed matroids}

Because of Theorem 3.4, we shall need to work on $M / \ell$. At the same time, we also need to keep track of all information on $M$. One way to deal with this is to consider a representation of $M$ on $M / \ell$. A signed matroid is a pair $(N, \Sigma)$, where $N$ is a binary matroid on $E$ and $\Sigma$ is a subset of $E$. A subset $X \subseteq E$ is called $\Sigma$-odd or $\Sigma$-even if $|X \cap \Sigma|$ is odd or even, respectively. The following simple proposition links the $\ell$-port of $M$ with the set of odd circuits of $M / \ell$. We omit its proof since it follows easily from the fact that, in a binary matroid, the intersection of any circuit and any cocircuit must have an even cardinality (see 9.1.2 in [13]). Recall that a cocycle is a disjoint union of cocircuits.

Proposition 3.1 Let $M$ be a binary matroid on $E \cup\{\ell\}$, where $\ell \notin E$. If $\Sigma \subseteq E$ such that $\Sigma \cup\{\ell\}$ is a cocycle of $M$, then the $\ell$-port of $M$ is exactly the set of $\Sigma$-odd circuits of $M / \ell$.

Let $(N, \Sigma)$ be a signed matroid. We denote its set of $\Sigma$-odd circuits by $\mathcal{O}_{N, \Sigma}$. If $N$ is the 1-sum of two matroids $N_{1}$ and $N_{2}$, then it is clear that $\mathcal{O}_{N, \Sigma}$ is the disjoint union of $\mathcal{O}_{N_{1}, \Sigma \cap E\left(N_{1}\right)}$ and $\mathcal{O}_{N_{2}, \Sigma \cap E\left(N_{2}\right)}$. The situation is more complicated if $N$ is the 2-sum of two matroids. The following lemma is what we shall need to deal with this scenario. Let $\left(E_{1}, E_{2}\right)$ be a 2-separation of $N$ and let $N_{1}, N_{2}$ be the two matroids induced by $\left(E_{1}, E_{2}\right)$.

Lemma 3.2 Let $C \in \mathcal{O}_{N, \Sigma}$ with $C \cap E_{1} \neq \emptyset \neq C \cap E_{2}$. If $C \cap E_{2}$ is $\Sigma$-even, then $\mathcal{O}_{N_{1}, \Sigma \cap E_{1}}$ is a minor of $\mathcal{O}_{N, \Sigma}$. If $C \cap E_{2}$ is $\Sigma$-odd, then $\mathcal{O}_{N_{1},\left(\Sigma \cap E_{1}\right) \cup\{p\}}$ is a minor of $\mathcal{O}_{N, \Sigma}$.

Proof. Let $C_{2}=C \cap E_{2}$. Then $C_{2} \cup\{p\}$ is a circuit of $N_{2}$. It follows that $N^{\prime}=N \backslash\left(E_{2}-C_{2}\right)$ is the 2-sum of $N_{1}$ and $C_{2} \cup\{p\}$ (see 7.1.15 in [13]). Moreover, $\mathcal{O}_{N^{\prime}, \Sigma^{\prime}}=\mathcal{O}_{N, \Sigma} \backslash\left(E_{2}-C_{2}\right)$, where $\Sigma^{\prime}=\Sigma-\left(E_{2}-C_{2}\right)$. Let $X$ be a maximal subset of $C_{2} \cap \Sigma$ such that $|X|$ is even. Then $\mathcal{O}_{N^{\prime}, \Sigma^{\prime}}=\mathcal{O}_{N^{\prime}, \Sigma^{\prime \prime}}$, where $\Sigma^{\prime \prime}=\Sigma^{\prime}-X$. Notice that $\left|C_{2} \cap \Sigma^{\prime \prime}\right| \leq 1$ and $\left|C_{2} \cap \Sigma^{\prime \prime}\right| \equiv\left|C_{2} \cap \Sigma\right|(\bmod 2)$. Let $e \in C_{2}$ such that $e \in \Sigma^{\prime \prime}$ in case $C_{2} \cap \Sigma^{\prime \prime} \neq \emptyset$. Let $N^{\prime \prime}=N^{\prime} /\left(C_{2}-\{e\}\right)$. Then $N^{\prime \prime} \cong N_{1}$ and $\mathcal{O}_{N^{\prime \prime}, \Sigma^{\prime \prime}}=\mathcal{O}_{N^{\prime}, \Sigma^{\prime \prime}} /\left(C_{2}-\{e\}\right)=\mathcal{O}_{N, \Sigma} \backslash\left(E_{2}-C_{2}\right) /\left(C_{2}-\{e\}\right)$, which proves the lemma.

\section{Regular matroids}

In this section we prove a few results on regular matroids, which will be extended in later sections to all matroids in $\Im$ using Theorem 3.4. Our proof relies on results of Tutte on chain groups [22]. 
Let $U$ be a matrix whose columns are indexed by a set $E$. A nonzero vector $\boldsymbol{x} \in\{0, \pm 1\}^{E}$ is $U$-primitive if $U \boldsymbol{x}=\mathbf{0}$ and, for every $\boldsymbol{y} \in \mathbb{Z}^{E}$ with $U \boldsymbol{y}=\mathbf{0}, \operatorname{spt}(\boldsymbol{y})$ is not a proper subset of $\operatorname{spt}(\boldsymbol{x})$. A vector $\boldsymbol{x} \in \mathbb{Q}^{E}$ is said to conform to a vector $\boldsymbol{y} \in \mathbb{Q}^{E}$ if $\operatorname{spt}(\boldsymbol{x}) \subseteq \operatorname{spt}(\boldsymbol{y})$ and $\boldsymbol{x}(e) \boldsymbol{y}(e)>0$, for all $e \in \operatorname{spt}(\boldsymbol{x})$. The following result was proved in $[22]$.

Theorem 4.1 Let $M$ be a regular matroid and let $U$ be a totally unimodular matrix representing $M$.

(i) If $\boldsymbol{x}$ is $U$-primitive then $\operatorname{spt}(\boldsymbol{x})$ is a circuit of $M$;

(ii) If $C$ is a circuit of $M$ then $C=\operatorname{spt}(\boldsymbol{x})$ for some $U$-primitive vector $\boldsymbol{x}$;

(iii) If $\boldsymbol{x}$ is an integral nonzero vector with $U \boldsymbol{x}=\mathbf{0}$, then there exist $U$-primitive vectors $\boldsymbol{x}_{1}, \boldsymbol{x}_{2}, \ldots, \boldsymbol{x}_{m}$ conforming to $\boldsymbol{x}$ such that $\boldsymbol{x}=\boldsymbol{x}_{1}+\boldsymbol{x}_{2}+\ldots+\boldsymbol{x}_{m}$.

In the rest of this section, let $M$ be a regular matroid on $E \cup\{\ell\}$, where $\ell \notin E$. Let $U$ be a totally unimodular matrix representing $M$ and let $A$ be the $\mathcal{P}_{M, \ell^{-}} E$ incidence matrix. We first prove two technical lemmas. Recall that $\overline{\boldsymbol{x}}=(|\boldsymbol{x}(k)|: k \in K)$, for any $\boldsymbol{x}$ indexed by $K$.

Lemma 4.1 For any $\boldsymbol{\alpha} \in \mathbb{Q}_{+}^{\mathcal{P}_{M, \ell}}$, there exists $\boldsymbol{x} \in \mathbb{Q}^{E \cup\{\ell\}}$ such that $U \boldsymbol{x}=\mathbf{0}, \boldsymbol{x}(\ell)=\boldsymbol{\alpha}^{T} \mathbf{1}$, and $\left.\overline{\boldsymbol{x}}\right|_{E} \leq A^{T} \boldsymbol{\alpha}$.

Proof. Let the members of $\mathcal{P}_{M, \ell}$ be $P_{1}, P_{2}, \ldots, P_{n}$, which are ordered according to the order of the rows of A. By Theorem 4.1(ii), for each $i=1,2, \ldots, n$, there exists $U$-primitive vector $\boldsymbol{p}_{i}$ with $\operatorname{spt}\left(\boldsymbol{p}_{i}\right)=P_{i} \cup\{\ell\}$. By replacing $\boldsymbol{p}_{i}$ with $-\boldsymbol{p}_{i}$, if necessary, we may assume that $\boldsymbol{p}_{i}(\ell)=1$. Let $\boldsymbol{x}=\left[\boldsymbol{p}_{1}, \boldsymbol{p}_{2}, \ldots, \boldsymbol{p}_{n}\right] \boldsymbol{\alpha}$. Then $U \boldsymbol{x}=[\mathbf{0}, \mathbf{0}, \ldots, \mathbf{0}] \boldsymbol{\alpha}=\mathbf{0}, \boldsymbol{x}(\ell)=\boldsymbol{\alpha}^{T} \mathbf{1}$, and $\overline{\boldsymbol{x}} \leq\left[\overline{\boldsymbol{p}}_{1}, \overline{\boldsymbol{p}}_{2}, \ldots, \overline{\boldsymbol{p}}_{n}\right] \boldsymbol{\alpha}$. Notice that, when projected to $\mathbb{Q}^{E}$, the last inequality is exactly $\left.\overline{\boldsymbol{x}}\right|_{E} \leq A^{T} \boldsymbol{\alpha}$, and thus the lemma is proved.

Lemma 4.2 For any $\boldsymbol{x} \in \mathbb{Q}^{E \cup\{\ell\}}$ with $U \boldsymbol{x}=\mathbf{0}$, there exists $\boldsymbol{\alpha} \in \mathbb{Q}_{+}^{\mathcal{P}_{M, \ell}}$ such that $\boldsymbol{\alpha}^{T} \mathbf{1} \geq \boldsymbol{x}(\ell)$ and $A^{T} \boldsymbol{\alpha} \leq\left.\overline{\boldsymbol{x}}\right|_{E}$. Moreover, if $\boldsymbol{x}$ is integral, then so is $\boldsymbol{\alpha}$.

Proof. We first consider the case when $\boldsymbol{x}$ is integral. If $\boldsymbol{x}(\ell) \leq 0$, then $\boldsymbol{\alpha}=\mathbf{0}$ satisfies the requirements. Hence we may assume $\boldsymbol{x}(\ell)=k>0$. By Theorem 4.1(iii), there exist $U$-primitive vectors $\boldsymbol{x}_{1}, \boldsymbol{x}_{2}, \ldots, \boldsymbol{x}_{m}$ conforming to $\boldsymbol{x}$ such that $\boldsymbol{x}=\boldsymbol{x}_{1}+\boldsymbol{x}_{2}+\ldots+\boldsymbol{x}_{m}$. Without loss of generality, let $\boldsymbol{x}_{1}(\ell)=\boldsymbol{x}_{2}(\ell)=\ldots=\boldsymbol{x}_{k}(\ell)=1$ and $\boldsymbol{x}_{k+1}(\ell)=\boldsymbol{x}_{k+2}(\ell)=\ldots=\boldsymbol{x}_{m}(\ell)=0$. Then we deduce from Theorem 4.1(i) that each $\left.\overline{\boldsymbol{x}}_{i}^{T}\right|_{E}(1 \leq i \leq k)$ is a row of $A$. Consequently, $\left.\left(\overline{\boldsymbol{x}}_{1}+\overline{\boldsymbol{x}}_{2}+\ldots+\overline{\boldsymbol{x}}_{k}\right)\right|_{E}=A^{T} \boldsymbol{\alpha}$, for some $\boldsymbol{\alpha} \in \mathbb{Z}_{+}^{\mathcal{P}_{M, \ell}}$ with $\boldsymbol{\alpha}^{T} \mathbf{1}=k=\boldsymbol{x}(\ell)$. Since each $\boldsymbol{x}_{i}$ conforms to $\boldsymbol{x}$, it follows that $\overline{\boldsymbol{x}}=\overline{\boldsymbol{x}}_{1}+\overline{\boldsymbol{x}}_{2}+\ldots+\overline{\boldsymbol{x}}_{m}$. Therefore, $\left.\overline{\boldsymbol{x}}\right|_{E} \geq\left.\left(\overline{\boldsymbol{x}}_{1}+\overline{\boldsymbol{x}}_{2}+\ldots+\overline{\boldsymbol{x}}_{k}\right)\right|_{E}=A^{T} \boldsymbol{\alpha}$.

If $\boldsymbol{x}$ is not integral, we may choose a positive integer $d$ such that $\boldsymbol{x}^{\prime}=d \boldsymbol{x}$ is integral. It follows from the conclusion in the last paragraph that there exists $\boldsymbol{\alpha}^{\prime} \in \mathbb{Z}_{+}^{\mathcal{P}_{M, \ell}}$ such that $\left(\boldsymbol{\alpha}^{\prime}\right)^{T} \mathbf{1} \geq \boldsymbol{x}^{\prime}(\ell)$ and $A^{T} \boldsymbol{\alpha}^{\prime} \leq\left.\overline{\boldsymbol{x}}^{\prime}\right|_{E}$. Then it is clear that $\boldsymbol{\alpha}=\frac{1}{d} \boldsymbol{\alpha}^{\prime}$ satisfies the requirements of the lemma.

The next lemma is the first step for our main proof.

Lemma 4.3 If $M$ is a regular matroid on $E \cup\{\ell\}$, where $\ell \notin E$, then $\mathcal{P}_{M, \ell}$ is box-Mengerian.

Proof. Let $\boldsymbol{l} \in \mathbb{Q}^{E}, \boldsymbol{u} \in \mathbb{Q}^{E}$, and $\boldsymbol{w} \in \mathbb{Z}^{E}$. We need to show that

$$
\max \left\{\boldsymbol{\alpha}^{T} \mathbf{1}+\boldsymbol{\beta}^{T} \boldsymbol{l}-\boldsymbol{\gamma}^{T} \boldsymbol{u}: \boldsymbol{\alpha}^{T} A+\boldsymbol{\beta}^{T}-\boldsymbol{\gamma}^{T} \leq \boldsymbol{w}^{T}, \boldsymbol{\alpha}, \boldsymbol{\beta}, \boldsymbol{\gamma} \geq \mathbf{0}\right\}
$$


which is the dual of $\min \left\{\boldsymbol{w}^{T} \boldsymbol{x}: A \boldsymbol{x} \geq \mathbf{1}, \boldsymbol{x} \geq \mathbf{0}, \boldsymbol{u} \geq \boldsymbol{x} \geq \boldsymbol{l}\right\}$, has an integral optimal solution, assuming that it has an optimal solution. We consider

$$
\max \left\{\boldsymbol{x}(\ell)+\boldsymbol{\beta}^{T} \boldsymbol{l}-\boldsymbol{\gamma}^{T} \boldsymbol{u}: U \boldsymbol{x}=\mathbf{0},\left(\left.\boldsymbol{x}\right|_{E}\right)+\boldsymbol{\beta}-\boldsymbol{\gamma} \leq \boldsymbol{w},-\left(\left.\boldsymbol{x}\right|_{E}\right)+\boldsymbol{\beta}-\boldsymbol{\gamma} \leq \boldsymbol{w}, \boldsymbol{\beta}, \boldsymbol{\gamma} \geq \mathbf{0}\right\} .
$$

Notice that the two inequalities $\left(\left.\boldsymbol{x}\right|_{E}\right)+\boldsymbol{\beta}-\boldsymbol{\gamma} \leq \boldsymbol{w}$ and $-\left(\left.\boldsymbol{x}\right|_{E}\right)+\boldsymbol{\beta}-\boldsymbol{\gamma} \leq \boldsymbol{w}$ can be equivalently combined as a single inequality $\left(\left.\overline{\boldsymbol{x}}\right|_{E}\right)+\boldsymbol{\beta}-\boldsymbol{\gamma} \leq \boldsymbol{w}$. The following two observations are clear from the last two lemmas.

(i) If $(\boldsymbol{\alpha}, \boldsymbol{\beta}, \boldsymbol{\gamma})$ is a feasible solution to (4.1), then there exists $\boldsymbol{x}$ such that $\boldsymbol{x}(\ell)=\boldsymbol{\alpha}^{T} \mathbf{1}$ and $(\boldsymbol{x}, \boldsymbol{\beta}, \boldsymbol{\gamma})$ is a feasible solution to (4.2).

(ii) If $(\boldsymbol{x}, \boldsymbol{\beta}, \boldsymbol{\gamma})$ is a feasible solution to (4.2), then there exists $\boldsymbol{\alpha}$ such that $\boldsymbol{\alpha}^{T} \mathbf{1} \geq \boldsymbol{x}(\ell)$ and $(\boldsymbol{\alpha}, \boldsymbol{\beta}, \boldsymbol{\gamma})$ is a feasible solution to (4.1). Moreover, if $(\boldsymbol{x}, \boldsymbol{\beta}, \boldsymbol{\gamma})$ is integral, then so is $(\boldsymbol{\alpha}, \boldsymbol{\beta}, \boldsymbol{\gamma})$.

Since (4.1) has feasible solutions, (i) implies that (4.2) has feasible solutions too. It also implies that the maximum of (4.1) is at most that of (4.2). On the other hand, (ii) implies that the maximum of (4.2) is at most that of (4.1). Thus (4.2) has a finite maximum and its maximum is the same as that of (4.1). In addition, (ii) also implies that every integral optimal solution of (4.2) induces an integral optimal solution of (4.1). Therefore, we only need to show that (4.2) has an integral optimal solution.

Let $\left(\boldsymbol{x}_{0}, \boldsymbol{\beta}_{0}, \gamma_{0}\right)$ be an optimal solution of (4.2). Let $E^{+}=\left\{e \in E: \boldsymbol{x}_{0}(e) \geq 0\right\}$ and $E^{-}=E-E^{+}$. Let $v$ denote the expression $\boldsymbol{x}(\ell)+\boldsymbol{\beta}^{T} \boldsymbol{l}-\boldsymbol{\gamma}^{T} \boldsymbol{u}$. Then

$$
\begin{aligned}
& \max \left\{v: U \boldsymbol{x}=\mathbf{0},\left(\left.\boldsymbol{x}\right|_{E}\right)+\boldsymbol{\beta}-\boldsymbol{\gamma} \leq \boldsymbol{w},-\left(\left.\boldsymbol{x}\right|_{E}\right)+\boldsymbol{\beta}-\boldsymbol{\gamma} \leq \boldsymbol{w}, \boldsymbol{\beta}, \boldsymbol{\gamma} \geq \mathbf{0}\right\} \\
= & \max \left\{v: U \boldsymbol{x}=\mathbf{0},\left(\left.\boldsymbol{x}\right|_{E}\right)+\boldsymbol{\beta}-\boldsymbol{\gamma} \leq \boldsymbol{w},-\left(\left.\boldsymbol{x}\right|_{E}\right)+\boldsymbol{\beta}-\boldsymbol{\gamma} \leq \boldsymbol{w}, \boldsymbol{\beta}, \boldsymbol{\gamma},\left.\boldsymbol{x}\right|_{E^{+}},\left.(-\boldsymbol{x})\right|_{E^{-}} \geq \mathbf{0}\right\} \\
= & \max \left\{v: U \boldsymbol{x}=\mathbf{0},\left.(\boldsymbol{x}+\boldsymbol{\beta}-\boldsymbol{\gamma})\right|_{E^{+}} \leq\left.\boldsymbol{w}\right|_{E^{+}},\left.(-\boldsymbol{x}+\boldsymbol{\beta}-\boldsymbol{\gamma})\right|_{E^{-}} \leq\left.\boldsymbol{w}\right|_{E^{-}}, \boldsymbol{\beta}, \boldsymbol{\gamma},\left.\boldsymbol{x}\right|_{E^{+}},\left.(-\boldsymbol{x})\right|_{E^{-}} \geq \mathbf{0}\right\} .
\end{aligned}
$$

It follows from the totally unimodularity of $U$ that the coefficient matrix of (4.3) is also totally unimodular. Thus (4.3) has an integral optimal solution $\left(\boldsymbol{x}^{*}, \boldsymbol{\beta}^{*}, \boldsymbol{\gamma}^{*}\right)$. Since all feasible solutions to (4.3) are also feasible to (4.2), the above equalities imply that $\left(\boldsymbol{x}^{*}, \boldsymbol{\beta}^{*}, \boldsymbol{\gamma}^{*}\right)$ is an integral optimal solution of (4.2).

A flow is a pair $(\boldsymbol{f}, \boldsymbol{\alpha})$ such that $\boldsymbol{\alpha} \geq \mathbf{0}$ and $\boldsymbol{f}=A^{T} \boldsymbol{\alpha}$. The next two lemmas are the base cases for more general results, which we shall use to analyze feasible solutions of the LP problem in our consideration.

Lemma 4.4 For any flow $(\boldsymbol{f}, \boldsymbol{\alpha})$ and any circuit $C$ of $M / \ell$ with $C \subseteq \operatorname{spt}(\boldsymbol{f})$, there exist $t \in\{0,1\}$ and $\chi \in\{0, \pm 1\}^{E}$ that satisfy the following properties:

(i) $\operatorname{spt}(\chi)=C$;

(ii) for any $\varepsilon \in \mathbb{Q}_{+}$with $\varepsilon<\frac{1}{2} \min _{e \in C} \boldsymbol{f}(e)$, there exist flows $\left(\boldsymbol{f}_{+}, \boldsymbol{\alpha}_{+}\right)$and $\left(\boldsymbol{f}_{-}, \boldsymbol{\alpha}_{-}\right)$such that $\boldsymbol{f}_{ \pm} \leq \boldsymbol{f} \pm \varepsilon \boldsymbol{\chi}$ and $\boldsymbol{\alpha}_{ \pm}^{T} \mathbf{1} \geq \boldsymbol{\alpha}^{T} \mathbf{1} \pm t \varepsilon$.

Proof. By Lemma 4.1, there exists $\boldsymbol{x} \in \mathbb{Q}^{E \cup\{\ell\}}$ such that $U \boldsymbol{x}=\mathbf{0}, \boldsymbol{x}(\ell)=\boldsymbol{\alpha}^{T} \mathbf{1}$, and $\left.\overline{\boldsymbol{x}}\right|_{E} \leq \boldsymbol{f}$. Let $D$ be the circuit of $M$ with $C \subseteq D \subseteq C \cup\{\ell\}$. By Theorem 4.1(ii), there exists a $U$-primitive vector $\boldsymbol{d}$ such that $\operatorname{spt}(\boldsymbol{d})=D$ and $\boldsymbol{d}(\ell) \geq 0$. Let $t=\boldsymbol{d}(\ell)$. We define $\boldsymbol{\chi} \in\{0, \pm 1\}^{E}$ as follows: $\chi(e)=0$ if $e \notin C, \boldsymbol{\chi}(e)=1$ if $e \in C$ and $\boldsymbol{x}(e) \boldsymbol{d}(e) \geq 0$, and $\boldsymbol{\chi}(e)=-1$ if $e \in C$ and $\boldsymbol{x}(e) \boldsymbol{d}(e)<0$. Clearly, (i) is satisfied.

For the given $\varepsilon$, let $\boldsymbol{y}_{ \pm}=\boldsymbol{x} \pm \varepsilon \boldsymbol{d}$. By applying Lemma 4.2 to $\boldsymbol{y}_{ \pm}$we get flows $\left(\boldsymbol{f}_{ \pm}, \boldsymbol{\alpha}_{ \pm}\right)$such that $\boldsymbol{\alpha}_{ \pm}^{T} \mathbf{1} \geq \boldsymbol{y}_{ \pm}(\ell)$ and $\boldsymbol{f}_{ \pm} \leq\left.\overline{\boldsymbol{y}}_{ \pm}\right|_{E}$. Notice that $\boldsymbol{y}_{ \pm}(\ell)=\boldsymbol{x}(\ell) \pm \varepsilon \boldsymbol{d}(\ell)=\boldsymbol{\alpha}^{T} \mathbf{1} \pm t \varepsilon$, so $\boldsymbol{\alpha}_{ \pm}^{T} \mathbf{1} \geq \boldsymbol{\alpha}^{T} \mathbf{1} \pm t \varepsilon$ holds. For the other inequality in (ii), our choice of $\boldsymbol{\alpha}_{ \pm}$and our definition of $\boldsymbol{y}_{ \pm}$imply that we only need to verify $|\boldsymbol{x}(e) \pm \varepsilon \boldsymbol{d}(e)| \leq \boldsymbol{f}(e) \pm \varepsilon \boldsymbol{\chi}(e)$, for all $e \in E$. If $e \notin C$, then $\boldsymbol{d}(e)=\boldsymbol{\chi}(e)=0$, so the inequality follows from 
our choice of $\boldsymbol{x}$. For each $e \in C$, we consider two cases: $|\boldsymbol{x}(e) \pm \varepsilon \boldsymbol{d}(e)|=|\boldsymbol{x}(e)|+\varepsilon$ or || $\boldsymbol{x}(e)|-\varepsilon|$. In the second case,

$$
|\boldsymbol{x}(e) \pm \varepsilon \boldsymbol{d}(e)|=\max \{|\boldsymbol{x}(e)|-\varepsilon, \varepsilon-|\boldsymbol{x}(e)|\} \leq \max \{|\boldsymbol{x}(e)|-\varepsilon, \varepsilon\} \leq \boldsymbol{f}(e)-\varepsilon \leq \boldsymbol{f}(e) \pm \varepsilon \boldsymbol{\chi}(e),
$$

where the second inequality follows from our choice of $\boldsymbol{x}$ and the restriction on $\varepsilon$. For the first case, let us also assume that the second case does not occur and thus $\boldsymbol{x}(e) \neq 0$. Then

$$
|\boldsymbol{x}(e) \pm \varepsilon \boldsymbol{d}(e)|=|\boldsymbol{x}(e)|+\varepsilon \leq \boldsymbol{f}(e)+\varepsilon=\boldsymbol{f}(e) \pm \varepsilon \boldsymbol{\chi}(e),
$$

where the last equality follows from the assumption $|\boldsymbol{x}(e) \pm \varepsilon \boldsymbol{d}(e)|=|\boldsymbol{x}(e)|+\varepsilon$.

For any two flows $(\boldsymbol{f}, \boldsymbol{\alpha})$ and $\left(\boldsymbol{f}^{\prime}, \boldsymbol{\alpha}^{\prime}\right)$, we write $\left(\boldsymbol{f}^{\prime}, \boldsymbol{\alpha}^{\prime}\right) \preceq(\boldsymbol{f}, \boldsymbol{\alpha})$ if $\boldsymbol{f}^{\prime} \leq \boldsymbol{f}$ and $\left(\boldsymbol{\alpha}^{\prime}\right)^{T} \mathbf{1} \geq \boldsymbol{\alpha}^{T} \mathbf{1}$. We call a flow $(\boldsymbol{f}, \boldsymbol{\alpha})$ minimal if $\boldsymbol{f}^{\prime}=\boldsymbol{f}$ and $\left(\boldsymbol{\alpha}^{\prime}\right)^{T} \mathbf{1}=\boldsymbol{\alpha}^{T} \mathbf{1}$ hold for all flows $\left(\boldsymbol{f}^{\prime}, \boldsymbol{\alpha}^{\prime}\right) \preceq(\boldsymbol{f}, \boldsymbol{\alpha})$. For any $\boldsymbol{x} \in \mathbb{Q}^{E}$, a subset $E^{\prime} \subseteq E$ is called $\boldsymbol{x}$-fractional if $\boldsymbol{x}(e) \notin \mathbb{Z}$, for every $e \in E^{\prime}$.

Lemma 4.5 If $(\boldsymbol{f}, \boldsymbol{\alpha})$ is a minimal flow and $e$ is an element of $M / \ell$ such that $\boldsymbol{f}(e)$ is not integral, then $M / \ell$ has an $\boldsymbol{f}$-fractional circuit $C$ that contains e.

Let us make a preparation before presenting a proof of this lemma.

Lemma 4.6 Let $N$ be a matroid on $F$, let $W$ be a totally unimodular matrix representing $N$, and let e $\in F$ and $\boldsymbol{x} \in \mathbb{Q}^{F}$ with $W \boldsymbol{x}=\mathbf{0}$. Then

(i) There is a totally unimodular matrix $W^{\prime}$ representing $N / e$ such that $W^{\prime} \boldsymbol{x}^{\prime}=\mathbf{0}$, where $\boldsymbol{x}^{\prime}=\left.\boldsymbol{x}\right|_{F-\{e\}}$;

(ii) $N$ has an $\boldsymbol{x}$-fractional circuit $C$ that contains $e$, if $\boldsymbol{x}(e)$ is not integral.

Proof. (i) Let $W_{e}$ be the column of $W$ that is indexed by $e$. If $e$ is a loop, then $W_{e}=\mathbf{0}$. In this case, let $W^{\prime}$ be obtained from $W$ by deleting $W_{e}$. Then $W^{\prime}$ satisfies the requirement since $N / e=N \backslash e$. If $e$ is not a loop, we extend it into a basis $B$. Let $W_{B}$ be the submatrix of $W$ that corresponds to $B$. Then $W_{B}^{-1} W$ is another totally unimodular matrix representing $N$ (see 2.2.20 in [13]). Let $W^{\prime}$ be obtained from $W_{B}^{-1} W$ by deleting the row and column indexed by $e$. Then $W^{\prime}$ satisfies the requirements (see 3.2.6 in [13]).

(ii) Suppose for a contradiction that the result is false for some $N$ and $\boldsymbol{x}$ with $|F|$ minimum. If $\boldsymbol{x}\left(e^{\prime}\right)$ is integral for all $e^{\prime} \in F-\{e\}$, then $W_{e}=\mathbf{0}$, as $W \boldsymbol{x}=\mathbf{0}$ and $W$ is an integral matrix. This is a contradiction because $C=\{e\}$ would be the required circuit. Therefore, we may assume that there exists $e^{\prime} \in F-\{e\}$ with $\boldsymbol{x}\left(e^{\prime}\right) \notin \mathbb{Z}$. By (i), there is a totally unimodular matrix $W^{\prime}$ representing $N / e^{\prime}$ such that $W^{\prime}\left(\left.\boldsymbol{x}\right|_{F-\left\{e^{\prime}\right\}}\right)=\mathbf{0}$. Then we deduce from the minimality of $|F|$ that $N / e^{\prime}$ has an $\boldsymbol{x}^{\prime}$-fractional circuit $C \ni e$. This means that either $C$ or $C \cup\{e\}$ is an $\boldsymbol{x}$-fractional circuit of $N$, which is a contradiction.

Proof of Lemma 4.5. By Lemma 4.1, there exists $\boldsymbol{x} \in \mathbb{Q}^{E \cup\{\ell\}}$ such that $U \boldsymbol{x}=\mathbf{0}, \boldsymbol{x}(\ell)=\boldsymbol{\alpha}^{T} \mathbf{1}$, and $\overline{\boldsymbol{y}} \leq \boldsymbol{f}$, where $\boldsymbol{y}=\left.\boldsymbol{x}\right|_{E}$. Then by Lemma 4.2 , there exists a flow $\left(\boldsymbol{f}^{\prime}, \boldsymbol{\alpha}^{\prime}\right)$ such that $\left(\boldsymbol{\alpha}^{\prime}\right)^{T} \mathbf{1} \geq \boldsymbol{x}(\ell)$ and $\boldsymbol{f}^{\prime} \leq \overline{\boldsymbol{y}}$. By the minimality of $(\boldsymbol{f}, \boldsymbol{\alpha})$, we have $\boldsymbol{f}=\overline{\boldsymbol{y}}$ (we do not need the fact $\left(\boldsymbol{\alpha}^{\prime}\right)^{T} \mathbf{1}=\boldsymbol{\alpha}^{T} \mathbf{1}$ here). By Lemma 4.6(i), there exists a totally unimodular matrix $U^{\prime}$ representing $M / \ell$ such that $U^{\prime} \boldsymbol{y}=\mathbf{0}$. Then by Lemma 4.6(ii), $M / \ell$ has a $\boldsymbol{y}$-fractional circuit $C \ni e$. Since $\boldsymbol{f}=\overline{\boldsymbol{y}}$, it follows that $C$ is $\boldsymbol{f}$-fractional, as required. 


\section{Flows}

The concept of flow introduced in the last section was for regular matroids. It can easily be extended to any signed matroid $(N, \Sigma)$. Let $A$ be the $\mathcal{O}_{N, \Sigma^{-}} E$ incidence matrix, where $E=E(N)$. Then a flow is a pair $(\boldsymbol{f}, \boldsymbol{\alpha})$ such that $\boldsymbol{\alpha} \geq \mathbf{0}$ and $\boldsymbol{f}=A^{T} \boldsymbol{\alpha}$. In case $N=M / \ell$, for a regular matroid $M$, such that $\Sigma \cup\{\ell\}$ is a cocycle of $M$, then Proposition 3.1 guarantees that this extension is consistent with our definition given in the last section.

The goal of this section is to generalize Lemma 4.4 and Lemma 4.5 from regular matroids to matroids in $\Im$. These results will be used in the next section to analyze feasible solutions to our LP problem. Our proof will use Theorem 3.4, which allows us to construct the required objects from parts that are obtained on regular matroids.

\subsection{Composition and decomposition}

Throughout this subsection, let $N$ be a connected binary matroid on $E$ and let $\Sigma \subseteq E$. Let $A$ be the $\mathcal{O}_{N, \Sigma}-E$ incidence matrix. Suppose $\left(E_{1}, E_{2}\right)$ is a 2-separation of $N$. For $i=1,2$, let $\mathcal{O}_{N, \Sigma}\left(E_{i}\right)$ denote the set of $\Sigma$-odd circuits $C$ with $C \subseteq E_{i}$. Let $\mathcal{O}_{N, \Sigma}\left(E_{1}, E_{2}\right)=\mathcal{O}_{N, \Sigma}-\mathcal{O}_{N, \Sigma}\left(E_{1}\right)-\mathcal{O}_{N, \Sigma}\left(E_{2}\right)$, which is the set of $\Sigma$-odd circuits that meet both $E_{1}$ and $E_{2}$. We may drop the subscripts when there is no confusion. Two members $C_{1}, C_{2} \in \mathcal{O}\left(E_{1}, E_{2}\right)$ are said to cross at $\left(E_{1}, E_{2}\right)$, if both $C_{1} \cap E_{1}$ and $C_{2} \cap E_{2}$ are $\Sigma$-odd. A flow $(\boldsymbol{f}, \boldsymbol{\alpha})$ is called cross-free at $\left(E_{1}, E_{2}\right)$ if no two members in $\mathcal{O}\left(E_{1}, E_{2}\right) \cap \operatorname{spt}(\boldsymbol{\alpha})$ cross. We shall call a flow $\left(\boldsymbol{f}^{\prime}, \boldsymbol{\alpha}^{\prime}\right)$ tight at $\left(E_{1}, E_{2}\right)$, with respect to a given flow $(\boldsymbol{f}, \boldsymbol{\alpha})$, if it is an optimal solution to the problem $\min \left\{\boldsymbol{\alpha}^{\prime}\left(\mathcal{O}\left(E_{1}, E_{2}\right)\right):\left(\boldsymbol{f}^{\prime}, \boldsymbol{\alpha}^{\prime}\right) \preceq(\boldsymbol{f}, \boldsymbol{\alpha})\right\}$. Notice that this problem must have an optimal solution since $\boldsymbol{\alpha}^{\prime} \geq \mathbf{0}$. The next lemma says that such a tight flow must be cross-free.

Lemma 5.1 For any 2-separation $\left(E_{1}, E_{2}\right)$ and any flow $(\boldsymbol{f}, \boldsymbol{\alpha})$, if $\left(\boldsymbol{f}^{\prime}, \boldsymbol{\alpha}^{\prime}\right)$ is tight at $\left(E_{1}, E_{2}\right)$ with respect to $(\boldsymbol{f}, \boldsymbol{\alpha})$, then $\left(\boldsymbol{f}^{\prime}, \boldsymbol{\alpha}^{\prime}\right)$ is cross-free at $\left(E_{1}, E_{2}\right)$.

Proof. Suppose there exist $C_{1}, C_{2} \in \mathcal{O}\left(E_{1}, E_{2}\right) \cap \operatorname{spt}\left(\boldsymbol{\alpha}^{\prime}\right)$ that cross. Let $N_{1}$ and $N_{2}$ be the two matroids induced by $\left(E_{1}, E_{2}\right)$. Then, for $i, j \in\{1,2\}, C_{i, j}=\left(C_{i} \cap E_{j}\right) \cup\{p\}$ is a circuit of $N_{j}$. Notice that $C_{1,1}$ and $C_{2,2}$ are $\Sigma$-odd while $C_{1,2}$ and $C_{2,1}$ are $\Sigma$-even, as $C_{1}$ and $C_{2}$ cross. It follows that the symmetric differences $C_{1,1} \Delta C_{2,1}$ and $C_{1,2} \Delta C_{2,2}$ are both $\Sigma$-odd. Since $N_{i}(i=1,2)$ is binary, $C_{1, i} \Delta C_{2, i}$ is a disjoint union of circuits of $N_{i} \backslash p$, which implies that at least one of these circuits $D_{i}$ is $\Sigma$-odd. Clearly, $D_{i} \in \mathcal{O}\left(E_{i}\right)$. Moreover, if we use $R_{C}$ to denote the row of $A$ that is indexed by $C \in \mathcal{O}_{M, \Sigma}$, then $R_{D_{1}}+R_{D_{2}} \leq R_{C_{1}}+R_{C_{2}}$.

Let $\delta=\min \left\{\boldsymbol{\alpha}^{\prime}\left(C_{1}\right), \boldsymbol{\alpha}^{\prime}\left(C_{2}\right)\right\}$. We define $\boldsymbol{\alpha}^{\prime \prime} \in \mathbb{Q}^{\mathcal{O}_{M, \Sigma}}$ with $\boldsymbol{\alpha}^{\prime \prime}\left(C_{i}\right)=\boldsymbol{\alpha}^{\prime}\left(C_{i}\right)-\delta$ and $\boldsymbol{\alpha}^{\prime \prime}\left(D_{i}\right)=\boldsymbol{\alpha}^{\prime}\left(D_{i}\right)+\delta$, for $i=1,2$, and $\boldsymbol{\alpha}^{\prime \prime}(C)=\boldsymbol{\alpha}^{\prime}(C)$, for all other $C \in \mathcal{O}_{M, \Sigma}$. The choice of $\delta$ implies $\boldsymbol{\alpha}^{\prime \prime} \geq \mathbf{0}$ and so $\left(\boldsymbol{f}^{\prime \prime}, \boldsymbol{\alpha}^{\prime \prime}\right)$ is a flow. It is routine to verify that $\left(\boldsymbol{\alpha}^{\prime \prime}\right)^{T} \mathbf{1}=\left(\boldsymbol{\alpha}^{\prime}\right)^{T} \mathbf{1}$ and $\boldsymbol{f}^{\prime \prime}=\boldsymbol{f}^{\prime}+\delta\left(R_{D_{1}}+R_{D_{2}}-R_{C_{1}}-R_{C_{2}}\right) \leq \boldsymbol{f}^{\prime}$. However, $\boldsymbol{\alpha}^{\prime \prime}\left(\mathcal{O}\left(E_{1}, E_{2}\right)\right)=\boldsymbol{\alpha}^{\prime}\left(\mathcal{O}\left(E_{1}, E_{2}\right)\right)-2 \delta$, contradicting the minimality of $\boldsymbol{\alpha}^{\prime}\left(\mathcal{O}\left(E_{1}, E_{2}\right)\right)$. Therefore, $\left(\boldsymbol{f}^{\prime}, \boldsymbol{\alpha}^{\prime}\right)$ is indeed cross-free at $\left(E_{1}, E_{2}\right)$, which proves the lemma.

Let $\left(E_{1}, E_{2}\right)$ be a 2-separation and let $N_{1}, N_{2}$ be the two matroids induced by $\left(E_{1}, E_{2}\right)$. Let flow $(\boldsymbol{f}, \boldsymbol{\alpha})$ be cross-free at $\left(E_{1}, E_{2}\right)$. Without loss of generality, we always assume that $C \cap E_{1}$ is $\Sigma$-odd for all $C \in$ $\mathcal{O}\left(E_{1}, E_{2}\right) \cap \operatorname{spt}(\boldsymbol{\alpha})$. Let $\Sigma_{1}=\Sigma \cap E_{1}$ and $\Sigma_{2}=\left(\Sigma \cap E_{2}\right) \cup\{p\}$. We shall refer to $\left(N_{1}, \Sigma_{1}\right)$ and $\left(N_{2}, \Sigma_{2}\right)$ as signed matroids induced by $\left(E_{1}, E_{2}\right)$. Notice that $\mathcal{O}_{N, \Sigma}\left(E_{1}\right) \subseteq \mathcal{O}_{N_{1}, \Sigma_{1}}, \mathcal{O}_{N, \Sigma}\left(E_{2}\right) \subseteq \mathcal{O}_{N_{2}, \Sigma_{2}}$, and each $C \in \mathcal{O}_{N, \Sigma}\left(E_{1}, E_{2}\right)$ with $C \cap E_{1} \Sigma$-odd can be partitioned into $C_{1}, C_{2}$ such that $C_{1} \cup\{p\} \in \mathcal{O}_{N_{1}, \Sigma_{1}}$ and $C_{2} \cup\{p\} \in \mathcal{O}_{N_{2}, \Sigma_{2}}$. In fact, $\mathcal{O}_{N_{1}, \Sigma_{1}}$ and $\mathcal{O}_{N_{2}, \Sigma_{2}}$ do not have any other members. 
To decompose $(\boldsymbol{f}, \boldsymbol{\alpha})$ into flows on $\left(N_{1}, \Sigma_{1}\right)$ and $\left(N_{2}, \Sigma_{2}\right)$, we naturally define $\boldsymbol{\alpha}_{i} \in \mathbb{Q}_{+}^{\mathcal{O}_{N_{i}, \Sigma_{i}}}(i=1,2)$ with

$$
\boldsymbol{\alpha}_{i}(C)= \begin{cases}\boldsymbol{\alpha}(C) & \text { if } C \in \mathcal{O}_{N, \Sigma}\left(E_{i}\right) \\ \sum\left\{\boldsymbol{\alpha}(D): D \in \mathcal{O}_{N, \Sigma}\left(E_{1}, E_{2}\right), D \cap E_{i}=C\right\} & \text { if } C \in \mathcal{O}_{N_{i}, \Sigma_{i}}-\mathcal{O}_{N, \Sigma}\left(E_{i}\right)\end{cases}
$$

The two flows $\left(\boldsymbol{f}_{1}, \boldsymbol{\alpha}_{1}\right)$ and $\left(\boldsymbol{f}_{2}, \boldsymbol{\alpha}_{2}\right)$ will be referred to as components of $(\boldsymbol{f}, \boldsymbol{\alpha})$ with respect to $\left(E_{1}, E_{2}\right)$. Recall that $\mathcal{O}\left(E_{1}, E_{2}\right)=\mathcal{O}_{N, \Sigma}\left(E_{1}, E_{2}\right)$ denotes the set of $\Sigma$-odd circuits that meet both $E_{1}$ and $E_{2}$ and that $\boldsymbol{\alpha}\left(\mathcal{O}\left(E_{1}, E_{2}\right)\right)$ is the summation of $\boldsymbol{\alpha}(C)$ over all $C \in \mathcal{O}\left(E_{1}, E_{2}\right)$. It is straightforward to verify that they satisfy the following.

Lemma 5.2 For $i=1,2,\left.\boldsymbol{f}_{i}\right|_{E_{i}}=\left.\boldsymbol{f}\right|_{E_{i}}$ and $\boldsymbol{f}_{i}(p)=\boldsymbol{\alpha}\left(\mathcal{O}\left(E_{1}, E_{2}\right)\right)$. Moreover, $\boldsymbol{\alpha}_{1}^{T} \mathbf{1}+\boldsymbol{\alpha}_{2}^{T} \mathbf{1}=\boldsymbol{\alpha}^{T} \mathbf{1}+$ $\boldsymbol{\alpha}\left(\mathcal{O}\left(E_{1}, E_{2}\right)\right)$.

Let $E_{i}, N_{i}$, and $\Sigma_{i}(i=1,2)$ be the same as above. Conversely, suppose $\left(\boldsymbol{f}_{i}, \boldsymbol{\alpha}_{i}\right)$ is a flow of $\left(N_{i}, \Sigma_{i}\right)$, for $i=1,2$. We explain how to compose these two to get a flow $(\boldsymbol{f}, \boldsymbol{\alpha})$ of $(N, \Sigma)$. Naturally, we set $\boldsymbol{\alpha}(C)=\boldsymbol{\alpha} i(C)$ if $p \notin C \in \mathcal{O}_{N_{i}, \Sigma_{i}}$. To define $\boldsymbol{\alpha}(C)$ for all $C \in \mathcal{O}_{N, \Sigma}\left(E_{1}, E_{2}\right)$, we consider $\mathcal{O}_{i}(i=1,2)$, which consist of all $C_{i} \in \mathcal{O}_{N_{i}, \Sigma_{i}}$ with $C_{i} \ni p$. We pair members of $\mathcal{O}_{1}$ with members of $\mathcal{O}_{2}$ in an "arbitrary" way, as detailed in the following algorithm:

Step 1: Set $\boldsymbol{a}=\mathbf{0} \in \mathbb{Q}_{+}^{\mathcal{O}\left(E_{1}, E_{2}\right)}, \boldsymbol{a}_{1}=\left.\boldsymbol{\alpha}_{1}\right|_{\mathcal{O}_{1}}$, and $\boldsymbol{a}_{2}=\left.\boldsymbol{\alpha}_{2}\right|_{\mathcal{O}_{2}}$.

Step 2: While there exists $C_{1} \in \mathcal{O}_{1}$ with $\boldsymbol{a}_{1}\left(C_{1}\right) \neq 0$ and $C_{2} \in \mathcal{O}_{2}$ with $\boldsymbol{a}_{2}\left(C_{2}\right) \neq 0$, decrease $\boldsymbol{a}_{i}\left(C_{i}\right)$ $(i=1,2)$ by $\delta$ and increase $\boldsymbol{a}\left(\left(C_{1}-\{p\}\right) \cup\left(C_{2}-\{p\}\right)\right)$ by $\delta$, where $\delta=\min \left\{\boldsymbol{a}_{1}\left(C_{1}\right), \boldsymbol{a}_{2}\left(C_{2}\right)\right\}$.

Step 3: Set $\left.\boldsymbol{\alpha}\right|_{\mathcal{O}\left(E_{1}, E_{2}\right)}=\boldsymbol{a}$.

Flow $(\boldsymbol{f}, \boldsymbol{\alpha})$ will be referred to as the sum of $\left(\boldsymbol{f}_{1}, \boldsymbol{\alpha}_{1}\right)$ and $\left(\boldsymbol{f}_{2}, \boldsymbol{\alpha}_{2}\right)$. For any $\mathcal{C} \subseteq \mathcal{O}_{N_{i}, \Sigma_{i}}(i=1$ or 2$)$, let $\hat{\mathcal{C}}=\left(\mathcal{C}-\mathcal{O}_{i}\right) \cup\left\{C \in \mathcal{O}\left(E_{1}, E_{2}\right):\left(C \cap E_{i}\right) \cup\{p\} \in \mathcal{C}\right\}$. It is routine to verify that $(\boldsymbol{f}, \boldsymbol{\alpha})$ satisfies the following.

Lemma 5.3 Let $(\boldsymbol{f}, \boldsymbol{\alpha})$ be the sum of $\left(\boldsymbol{f}_{1}, \boldsymbol{\alpha}_{1}\right)$ and $\left(\boldsymbol{f}_{2}, \boldsymbol{\alpha}_{2}\right)$. Then $\left.\boldsymbol{f}\right|_{E_{1}} \leq\left.\boldsymbol{f}_{1}\right|_{E_{1}},\left.\boldsymbol{f}\right|_{E_{2}} \leq\left.\boldsymbol{f}_{2}\right|_{E_{2}}, \boldsymbol{\alpha}^{T} \mathbf{1}=$ $\boldsymbol{\alpha}_{1}^{T} \mathbf{1}+\boldsymbol{\alpha}_{2}^{T} \mathbf{1}-\max \left\{\boldsymbol{f}_{1}(p), \boldsymbol{f}_{2}(p)\right\}, \boldsymbol{\alpha}\left(\mathcal{O}\left(E_{1}, E_{2}\right)\right)=\min \left\{\boldsymbol{f}_{1}(p), \boldsymbol{f}_{2}(p)\right\}$, and $\boldsymbol{\alpha}(\hat{\mathcal{C}}) \leq \boldsymbol{\alpha}_{i}(\mathcal{C})\left(\forall \mathcal{C} \subseteq \mathcal{O}_{N_{i}, \Sigma_{i}}, i=\right.$ $1,2)$. Moreover, $\boldsymbol{\alpha}$ is integral if both $\boldsymbol{\alpha}_{1}$ and $\boldsymbol{\alpha}_{2}$ are.

\subsection{Generalizations}

In this subsection, we present generalizations of Lemma 4.4 and Lemma 4.5, assuming that $(M, \ell) \in \Im$, $N=M / \ell$ is connected, $E(N)=E, \Sigma \subseteq E$, and $\Sigma \cup\{\ell\}$ is a cocycle of $M$. For any 2-separation $\left(E_{1}, E_{2}\right)$ of $N$, a flow $(\boldsymbol{f}, \boldsymbol{\alpha})$ is called $\left(E_{1}, E_{2}\right)$-minimal if $\boldsymbol{f}^{\prime}=\boldsymbol{f}$ and $\left(\boldsymbol{\alpha}^{\prime}\right)^{T} \mathbf{1}=\boldsymbol{\alpha}^{T} \mathbf{1}$ hold for all flows $\left(\boldsymbol{f}^{\prime}, \boldsymbol{\alpha}^{\prime}\right) \preceq(\boldsymbol{f}, \boldsymbol{\alpha})$ with $\boldsymbol{\alpha}^{\prime}\left(\mathcal{O}\left(E_{1}, E_{2}\right)\right) \leq \boldsymbol{\alpha}\left(\mathcal{O}\left(E_{1}, E_{2}\right)\right)$. Notice that a minimal flow is $\left(E_{1}, E_{2}\right)$-minimal for every 2-separation $\left(E_{1}, E_{2}\right)$. A flow $(\boldsymbol{f}, \boldsymbol{\alpha})$ is called connected if $\boldsymbol{\alpha}^{\prime}\left(\mathcal{O}\left(E_{1}, E_{2}\right)\right)>0$ holds for all flows $\left(\boldsymbol{f}^{\prime}, \boldsymbol{\alpha}^{\prime}\right) \preceq(\boldsymbol{f}, \boldsymbol{\alpha})$ and all 2-separations $\left(E_{1}, E_{2}\right)$ of $N$. We first prove a technical lemma, which will be used in both of our generalizations.

Lemma 5.4 Let $\left(E_{1}, E_{2}\right)$ be a 2-separation of $N$ and let $(\boldsymbol{f}, \boldsymbol{\alpha})$ be a connected flow that is $\left(E_{1}, E_{2}\right)$-minimal. Let $\left(\boldsymbol{f}^{\prime}, \boldsymbol{\alpha}^{\prime}\right)$ be tight at $\left(E_{1}, E_{2}\right)$ with respect to $(\boldsymbol{f}, \boldsymbol{\alpha})$. If $\left(\boldsymbol{f}_{1}^{\prime}, \boldsymbol{\alpha}_{1}^{\prime}\right)$ and $\left(\boldsymbol{f}_{2}^{\prime}, \boldsymbol{\alpha}_{2}^{\prime}\right)$ are components of $\left(\boldsymbol{f}^{\prime}, \boldsymbol{\alpha}^{\prime}\right)$ with respect to $\left(E_{1}, E_{2}\right)$, then both $\left(\boldsymbol{f}_{1}^{\prime}, \boldsymbol{\alpha}_{1}^{\prime}\right)$ and $\left(\boldsymbol{f}_{2}^{\prime}, \boldsymbol{\alpha}_{2}^{\prime}\right)$ are minimal and connected. 
Proof. By Lemma 5.1, $\left(\boldsymbol{f}^{\prime}, \boldsymbol{\alpha}^{\prime}\right)$ is cross-free at $\left(E_{1}, E_{2}\right)$ and thus $\left(\boldsymbol{f}_{1}^{\prime}, \boldsymbol{\alpha}_{1}^{\prime}\right)$ and $\left(\boldsymbol{f}_{2}^{\prime}, \boldsymbol{\alpha}_{2}^{\prime}\right)$ are well defined. Let $\left(N_{1}, \Sigma_{1}\right)$ and $\left(N_{2}, \Sigma_{2}\right)$ be the signed matroids induced by $\left(E_{1}, E_{2}\right)$. For $i=1,2$, let $\left(\boldsymbol{f}_{i}^{\prime \prime}, \boldsymbol{\alpha}_{i}^{\prime \prime}\right) \preceq\left(\boldsymbol{f}_{i}^{\prime}, \boldsymbol{\alpha}_{i}^{\prime}\right)$ be a flow of $\left(N_{i}, \Sigma_{i}\right)$. We need to show that $\boldsymbol{f}_{i}^{\prime \prime}=\boldsymbol{f}_{i}^{\prime},\left(\boldsymbol{\alpha}_{i}^{\prime \prime}\right)^{T} \mathbf{1}=\left(\boldsymbol{\alpha}_{i}^{\prime}\right)^{T} \mathbf{1}$, and $\boldsymbol{\alpha}_{i}^{\prime \prime}\left(\mathcal{O}_{N_{i}, \Sigma_{i}}(X, Y)\right)>0$, for every 2-separation $(X, Y)$ of $N_{i}$.

Let $\left(\boldsymbol{f}^{\prime \prime}, \boldsymbol{\alpha}^{\prime \prime}\right)$ be the sum of $\left(\boldsymbol{f}_{1}^{\prime \prime}, \boldsymbol{\alpha}_{1}^{\prime \prime}\right)$ and $\left(\boldsymbol{f}_{2}^{\prime \prime}, \boldsymbol{\alpha}_{2}^{\prime \prime}\right)$. Then we deduce from Lemma 5.3 and Lemma 5.2 that, for $i=1,2$,

$$
\left.\boldsymbol{f}^{\prime \prime}\right|_{E_{i}} \leq\left.\boldsymbol{f}_{i}^{\prime \prime}\right|_{E_{i}} \leq\left.\boldsymbol{f}_{i}^{\prime}\right|_{E_{i}}=\left.\boldsymbol{f}^{\prime}\right|_{E_{i}} \leq\left.\boldsymbol{f}\right|_{E_{i}},
$$

and so $\boldsymbol{f}^{\prime \prime} \leq \boldsymbol{f}$. Using the same lemmas,

$$
\begin{aligned}
\left(\boldsymbol{\alpha}^{\prime \prime}\right)^{T} \mathbf{1} & =\left(\boldsymbol{\alpha}_{1}^{\prime \prime}\right)^{T} \mathbf{1}+\left(\boldsymbol{\alpha}_{2}^{\prime \prime}\right)^{T} \mathbf{1}-\max \left\{\boldsymbol{f}_{1}^{\prime \prime}(p), \boldsymbol{f}_{2}^{\prime \prime}(p)\right\} \\
& \geq\left(\boldsymbol{\alpha}_{1}^{\prime}\right)^{T} \mathbf{1}+\left(\boldsymbol{\alpha}_{2}^{\prime}\right)^{T} \mathbf{1}-\max \left\{\boldsymbol{f}_{1}^{\prime}(p), \boldsymbol{f}_{2}^{\prime}(p)\right\} \\
& =\left(\boldsymbol{\alpha}^{\prime}\right)^{T} \mathbf{1}+\boldsymbol{\alpha}^{\prime}\left(\mathcal{O}\left(E_{1}, E_{2}\right)\right)-\max \left\{\boldsymbol{f}_{1}^{\prime}(p), \boldsymbol{f}_{2}^{\prime}(p)\right\} \\
& =\left(\boldsymbol{\alpha}^{\prime}\right)^{T} \mathbf{1} \geq \boldsymbol{\alpha}^{T} \mathbf{1},
\end{aligned}
$$

and also by the tightness of $\left(\boldsymbol{f}^{\prime}, \boldsymbol{\alpha}^{\prime}\right)$,

$$
\boldsymbol{\alpha}^{\prime \prime}\left(\mathcal{O}\left(E_{1}, E_{2}\right)\right)=\min \left\{\boldsymbol{f}_{1}^{\prime \prime}(p), \boldsymbol{f}_{2}^{\prime \prime}(p)\right\} \leq \min \left\{\boldsymbol{f}_{1}^{\prime}(p), \boldsymbol{f}_{2}^{\prime}(p)\right\}=\boldsymbol{\alpha}^{\prime}\left(\mathcal{O}\left(E_{1}, E_{2}\right)\right) \leq \boldsymbol{\alpha}\left(\mathcal{O}\left(E_{1}, E_{2}\right)\right) .
$$

Since $(\boldsymbol{f}, \boldsymbol{\alpha})$ is $\left(E_{1}, E_{2}\right)$-minimal, we must have $\boldsymbol{f}^{\prime \prime}=\boldsymbol{f}$ and $\left(\boldsymbol{\alpha}^{\prime \prime}\right)^{T} \mathbf{1}=\boldsymbol{\alpha}^{T} \mathbf{1}$. Thus we deduce from (5.1) that $\left.\boldsymbol{f}_{i}^{\prime \prime}\right|_{E_{i}}=\left.\boldsymbol{f}_{i}^{\prime}\right|_{E_{i}}$, and from (5.2) that $\left(\boldsymbol{\alpha}_{i}^{\prime \prime}\right)^{T} \mathbf{1}=\left(\boldsymbol{\alpha}_{i}^{\prime}\right)^{T} \mathbf{1}(i=1,2)$ and $\max \left\{\boldsymbol{f}_{1}^{\prime \prime}(p), \boldsymbol{f}_{2}^{\prime \prime}(p)\right\}=\boldsymbol{\alpha}^{\prime}\left(\mathcal{O}\left(E_{1}, E_{2}\right)\right)$. By Lemma 5.3 and the tightness of $\left(\boldsymbol{f}^{\prime}, \boldsymbol{\alpha}^{\prime}\right)$, we also have $\min \left\{\boldsymbol{f}_{1}^{\prime \prime}(p), \boldsymbol{f}_{2}^{\prime \prime}(p)\right\}=\boldsymbol{\alpha}^{\prime \prime}\left(\mathcal{O}\left(E_{1}, E_{2}\right)\right) \geq \boldsymbol{\alpha}^{\prime}\left(\mathcal{O}\left(E_{1}, E_{2}\right)\right)$. Therefore, for $i=1,2, \boldsymbol{f}_{i}^{\prime \prime}(p)=\boldsymbol{\alpha}^{\prime}\left(\mathcal{O}\left(E_{1}, E_{2}\right)\right)=\boldsymbol{f}_{i}^{\prime}(p)$, which implies $\boldsymbol{f}_{i}^{\prime \prime}=\boldsymbol{f}_{i}^{\prime}$, and thus $\left(\boldsymbol{f}_{i}^{\prime}, \boldsymbol{\alpha}_{i}^{\prime}\right)$ is minimal.

Next, suppose $(X, Y)$ is a 2-separation of $N_{i}(i=1,2)$ with $p \in Y$. We claim that $(X, E-X)$ is a 2-separation of $N$. To see this, let $N_{X}, N_{Y}$ be the two matroids induced by $(X, Y)$ and let $N_{Y}^{\prime}$ be the 2-sum of $N_{Y}$ and $N_{j}$, where $j \in\{1,2\}-\{i\}$. Then the definition of 2-sum implies that $N$ is the 2-sum of $N_{X}$ and $N_{Y}^{\prime}$, so $(X, E-X)$ is a 2-separation of $N$. Let $\mathcal{C}=\mathcal{O}_{N_{i}, \Sigma_{i}}(X, Y)$. Then its definition implies $\hat{\mathcal{C}}=\mathcal{O}_{N, \Sigma}(X, E-X)$. By Lemma 5.3 and the connectivity of $(\boldsymbol{f}, \boldsymbol{\alpha})$, we have $\boldsymbol{\alpha}_{i}^{\prime \prime}(\mathcal{C}) \geq \boldsymbol{\alpha}^{\prime \prime}(\hat{\mathcal{C}})>0$, which proves that $\left(\boldsymbol{f}_{i}^{\prime}, \boldsymbol{\alpha}_{i}^{\prime}\right)$ is connected.

Now we generalize Lemma 4.4.

Lemma 5.5 Suppose a connected flow $(\boldsymbol{f}, \boldsymbol{\alpha})$ is either minimal or $\left(E_{1}, E_{2}\right)$-minimal, for a 2-separation $\left(E_{1}, E_{2}\right)$ of $N$. Then for any circuit $C \in \mathcal{O}_{N, \Sigma}$ with $C \subseteq \operatorname{spt}(\boldsymbol{f})$, there exist $t \in \mathbb{Z}, \delta>0$, and $\boldsymbol{\chi} \in\{0, \pm 1\}^{E}$ that satisfy the following properties:

(i) $\operatorname{spt}(\boldsymbol{\chi})=C$;

(ii) for any $\varepsilon \in \mathbb{Q}_{+}$with $\varepsilon<\delta$, there exist flows $\left(\boldsymbol{f}_{+}, \boldsymbol{\alpha}_{+}\right)$and $\left(\boldsymbol{f}_{-}, \boldsymbol{\alpha}_{-}\right)$such that $\boldsymbol{f}_{ \pm} \leq \boldsymbol{f} \pm \varepsilon \boldsymbol{\chi}$ and $\boldsymbol{\alpha}_{ \pm}^{T} \mathbf{1} \geq \boldsymbol{\alpha}^{T} \mathbf{1} \pm$ te. Moreover, if $C \in \mathcal{O}\left(E_{1}, E_{2}\right)$, then $\left(\boldsymbol{f}_{ \pm}, \boldsymbol{\alpha}_{ \pm}\right)$can be chosen with the extra property that $\min \left\{\boldsymbol{\alpha}_{+}\left(\mathcal{O}\left(E_{1}, E_{2}\right)\right), \boldsymbol{\alpha}_{-}\left(\mathcal{O}\left(E_{1}, E_{2}\right)\right)\right\} \leq \boldsymbol{\alpha}\left(\mathcal{O}\left(E_{1}, E_{2}\right)\right)-\varepsilon$.

Proof. We prove by induction on $|E|$. If $N$ is 3-connected, by Theorem 3.4, $M$ is regular and so the result follows from Lemma 4.4. Therefore, we may proceed to the induction step and also assume that $N$ has a 2separation. Since a minimal flow is $\left(E_{1}, E_{2}\right)$-minimal, for every 2-separation $\left(E_{1}, E_{2}\right)$ of $N$, we may assume, 
without loss of generality, that $(\boldsymbol{f}, \boldsymbol{\alpha})$ is $\left(E_{1}, E_{2}\right)$-minimal. Let $\left(\boldsymbol{f}^{\prime}, \boldsymbol{\alpha}^{\prime}\right)$ be a flow tight at $\left(E_{1}, E_{2}\right)$, with respect to $(\boldsymbol{f}, \boldsymbol{\alpha})$. By Lemma $5.1,\left(\boldsymbol{f}^{\prime}, \boldsymbol{\alpha}^{\prime}\right)$ is cross-free at $\left(E_{1}, E_{2}\right)$. The connectedness and minimality of $(\boldsymbol{f}, \boldsymbol{\alpha})$ imply that $\left(\boldsymbol{f}^{\prime}, \boldsymbol{\alpha}^{\prime}\right)$ is also connected and $\left(E_{1}, E_{2}\right)$-minimal. Therefore, by the tightness of $\left(\boldsymbol{f}^{\prime}, \boldsymbol{\alpha}^{\prime}\right)$, we may assume, without loss of generality, that $\left(\boldsymbol{f}^{\prime}, \boldsymbol{\alpha}^{\prime}\right)=(\boldsymbol{f}, \boldsymbol{\alpha})$. Let $\left(\boldsymbol{f}_{1}, \boldsymbol{\alpha}_{1}\right)$ and $\left(\boldsymbol{f}_{2}, \boldsymbol{\alpha}_{2}\right)$ be the components of $(\boldsymbol{f}, \boldsymbol{\alpha})$ with respect to $\left(E_{1}, E_{2}\right)$. By Lemma 5.4 , they both are minimal and connected.

If $C \in \mathcal{O}\left(E_{1}, E_{2}\right)$, let $C_{i}=\left(C \cap E_{i}\right) \cup\{p\}$, for $i=1,2$. If $C \notin \mathcal{O}\left(E_{1}, E_{2}\right)$, let $C_{i}=C \cap E_{i}$, for $i=1,2$. By induction, there exist $t_{i}, \delta_{i}$, and $\chi_{i}$ that satisfy our induction hypothesis. We remark that, in the situation $C_{i}=\emptyset$, the choice $t_{i}=0, \delta_{i}=1$, and $\boldsymbol{\chi}_{i}=\mathbf{0}$ satisfy the requirements, as we may take $\left(\boldsymbol{f}_{i \pm}, \boldsymbol{\alpha}_{i \pm}\right)=\left(\boldsymbol{f}_{i}, \boldsymbol{\alpha}_{i}\right)$. By replacing $\boldsymbol{\chi}_{i}$ with $-\boldsymbol{\chi}_{i}$, if necessary, we assume that $\boldsymbol{\chi}_{i}(p) \geq 0$. Let $t_{0}=\boldsymbol{\chi}_{1}(p)=\boldsymbol{\chi}_{2}(p), t=t_{1}+t_{2}-t_{0}$, and $\delta=\min \left\{\delta_{1}, \delta_{2}\right\}$. Let $\chi \in\{0, \pm 1\}^{E}$ with $\left.\boldsymbol{\chi}\right|_{E_{i}}=\left.\chi_{i}\right|_{E_{i}}$, for $i=1,2$. Then (i) is clearly satisfied.

For any rational $\varepsilon$ with $0<\varepsilon<\delta$, let $\left(\boldsymbol{f}_{i \pm}, \boldsymbol{\alpha}_{i \pm}\right)$ be flows obtained from the induction hypothesis. Let $\left(\boldsymbol{f}_{ \pm}, \boldsymbol{\alpha}_{ \pm}\right)$be the sum of $\left(\boldsymbol{f}_{1 \pm}, \boldsymbol{\alpha}_{1 \pm}\right)$ and $\left(\boldsymbol{f}_{2 \pm}, \boldsymbol{\alpha}_{2 \pm}\right)$. We prove that they satisfy the requirements. First, $\left.\boldsymbol{f}_{ \pm}\right|_{E_{i}} \leq\left.\boldsymbol{f}_{i \pm}\right|_{E_{i}} \leq\left.\left(\boldsymbol{f}_{i} \pm \varepsilon \boldsymbol{\chi}_{i}\right)\right|_{E_{i}}=\left.(\boldsymbol{f} \pm \varepsilon \boldsymbol{\chi})\right|_{E_{i}}$, for $i=1,2$, so $\boldsymbol{f}_{ \pm} \leq \boldsymbol{f} \pm \varepsilon \boldsymbol{\chi}$. Next,

$$
\begin{aligned}
\boldsymbol{\alpha}_{ \pm}^{T} \mathbf{1} & =\boldsymbol{\alpha}_{1 \pm}^{T} \mathbf{1}+\boldsymbol{\alpha}_{2 \pm}^{T} \mathbf{1}-\max \left\{\boldsymbol{f}_{1 \pm}(p), \boldsymbol{f}_{2 \pm}(p)\right\} \\
& \geq \boldsymbol{\alpha}_{1}^{T} \mathbf{1}+\boldsymbol{\alpha}_{2}^{T} \mathbf{1} \pm\left(t_{1}+t_{2}\right) \varepsilon-\max \left\{\boldsymbol{f}_{1}(p) \pm t_{0} \varepsilon, \boldsymbol{f}_{2}(p) \pm t_{0} \varepsilon\right\} \\
& =\boldsymbol{\alpha}^{T} \mathbf{1} \pm\left(t_{1}+t_{2}-t_{0}\right) \varepsilon+\boldsymbol{\alpha}\left(\mathcal{O}\left(E_{1}, E_{2}\right)\right)-\max \left\{\boldsymbol{f}_{1}(p), \boldsymbol{f}_{2}(p)\right\} \\
& =\boldsymbol{\alpha}^{T} \mathbf{1} \pm t \varepsilon
\end{aligned}
$$

Finally, in case $C \in \mathcal{O}\left(E_{1}, E_{2}\right)$, we have

$$
\begin{aligned}
\min \left\{\boldsymbol{\alpha}_{+}\left(\mathcal{O}\left(E_{1}, E_{2}\right)\right), \boldsymbol{\alpha}_{-}\left(\mathcal{O}\left(E_{1}, E_{2}\right)\right)\right\} & =\min \left\{\boldsymbol{f}_{1 \pm}(p), \boldsymbol{f}_{2 \pm}(p)\right\} \\
& \leq \min \left\{\boldsymbol{f}_{1}(p) \pm \varepsilon, \boldsymbol{f}_{2}(p) \pm \varepsilon\right\}=\boldsymbol{\alpha}\left(\mathcal{O}\left(E_{1}, E_{2}\right)\right)-\varepsilon
\end{aligned}
$$

which completes our proof.

The next is a generalization of Lemma 4.5.

Lemma 5.6 If $(\boldsymbol{f}, \boldsymbol{\alpha})$ is a minimal and connected flow and $e$ is an element of $N$ such that $\boldsymbol{f}(e)$ is not integral, then $N$ has an $\boldsymbol{f}$-fractional circuit $C$ that contains $e$.

Proof. Our strategy is the same as that used in proving the last lemma. We prove by induction on $|E|$. If $N$ is 3-connected then $M$ is regular and so the result follows from Lemma 4.5. We proceed to the induction step and assume that $N$ has a 2-separation $\left(E_{1}, E_{2}\right)$ with, say, $e \in E_{1}$. Let $\left(\boldsymbol{f}^{\prime}, \boldsymbol{\alpha}^{\prime}\right)$ be a flow tight at this 2 -separation, with respect to $(\boldsymbol{f}, \boldsymbol{\alpha})$. The minimality of $(\boldsymbol{f}, \boldsymbol{\alpha})$ implies $\boldsymbol{f}^{\prime}=\boldsymbol{f}$, so we may assume, without loss of generality, that $\boldsymbol{\alpha}^{\prime}=\boldsymbol{\alpha}$ as well. By Lemma $5.1,(\boldsymbol{f}, \boldsymbol{\alpha})$ is cross-free at $\left(E_{1}, E_{2}\right)$. Thus we may consider $\left(\boldsymbol{f}_{1}, \boldsymbol{\alpha}_{1}\right)$ and $\left(\boldsymbol{f}_{2}, \boldsymbol{\alpha}_{2}\right)$, the components of $(\boldsymbol{f}, \boldsymbol{\alpha})$ with respect to $\left(E_{1}, E_{2}\right)$. By Lemma 5.4 , they both are minimal and connected. Let $\left(N_{1}, \Sigma_{1}\right)$ and $\left(N_{2}, \Sigma_{2}\right)$ be the signed matroids induced by $\left(E_{1}, E_{2}\right)$. By Lemma $5.2, \boldsymbol{f}_{1}(e)=\boldsymbol{f}(e)$, which is not integral, so we deduce from the induction hypothesis that $N_{1}$ has an $\boldsymbol{f}_{1}$-fractional circuit $C_{1}$ that contains $e$. If $C_{1} \not \nexists p$, then $C_{1}$ is a circuit of $N$ and thus $C=C_{1}$ is $\boldsymbol{f}$-fractional, as $\left.\boldsymbol{f}\right|_{E_{1}}=\left.\boldsymbol{f}_{1}\right|_{E_{1}}$. On the other hand, if $C_{1} \ni p$, then $\boldsymbol{f}_{1}(p)$ is not integral, which implies, by Lemma 5.2, that $\boldsymbol{f}_{2}(p)=\boldsymbol{f}_{1}(p)$ is not integral either. By induction, $N_{2}$ has an $\boldsymbol{f}_{2}$-fractional circuit $C_{2}$ that contains $p$. In this case, $C=\left(C_{1}-\{p\}\right) \cup\left(C_{2}-\{p\}\right)$ is a circuit of $N$, so it is $\boldsymbol{f}$-fractional, as $\left.\boldsymbol{f}\right|_{E_{i}}=\boldsymbol{f}_{i} \mid E_{i}(i=1,2)$. 


\section{Proving the main result}

Throughout this section, let $M$ be a matroid on $E \cup\{\ell\}$ with $\ell \notin E$. Let $N=M / \ell$ and let $\Sigma \subseteq E$ such that $\Sigma \cup\{\ell\}$ is a cocycle of $M$.

For any 0-1 matrix $A$, any rational vectors $\boldsymbol{l}$ and $\boldsymbol{u}$, and any integral vector $\boldsymbol{w}$, let $\operatorname{Max}(A, \boldsymbol{l}, \boldsymbol{u}, \boldsymbol{w})$ denote the LP problem expressed in (4.1). Our goal is to find, for every $A=A_{\mathcal{P}_{M, \ell}}$ with $(M, \ell) \in \Im$, an integral optimal solution of $\operatorname{Max}(A, \boldsymbol{l}, \boldsymbol{u}, \boldsymbol{w})$, when there is an optimal solution. The way we achieve this is to analyze potential minor minimal counterexamples $\mathcal{L}$. That is, $\mathcal{L}=\mathcal{P}_{M, \ell}$ is not box-Mengerian yet all its proper minors are. Equivalently, $\mathcal{P}_{M, \ell}$ is not box-Mengerian, but $\mathcal{P}_{M^{\prime}, \ell}$ is, for every proper minor $M^{\prime}$ of $M$ that uses $\ell$.

Throughout this section, we assume that $\mathcal{P}_{M, \ell}$ is a minor minimal counterexample and $A=A_{\mathcal{P}_{M, \ell}}$. We first prove that $(M, \ell) \in \Im$ and $N$ is connected, which guarantee that results proved in the last section can be used here. Next we prove that if $(\boldsymbol{\alpha}, \boldsymbol{\beta}, \boldsymbol{\gamma})$ is an optimal solution of $\operatorname{Max}(A, \boldsymbol{l}, \boldsymbol{u}, \boldsymbol{w})$ then $\boldsymbol{\alpha}\left(\mathcal{O}_{N, \Sigma}\left(E_{1}, E_{2}\right)\right)$ is not integral, for all 2-separations $\left(E_{1}, E_{2}\right)$ of $N$. Finally, we prove using lemmas established in the last section that, for any 2-separation $\left(E_{1}, E_{2}\right), \boldsymbol{\alpha}\left(\mathcal{O}_{N, \Sigma}\left(E_{1}, E_{2}\right)\right)$ is integral for at least one optimal solution $(\boldsymbol{\alpha}, \boldsymbol{\beta}, \boldsymbol{\gamma})$. This contradiction will eliminate all counterexamples and thus will prove the theorem.

We proceed by proving a sequence of lemmas. If $\ell$ is a loop or coloop in $M$, then $\mathcal{P}_{M, \ell}=\{\emptyset\}$ or $\emptyset$, respectively. By definition, they are box-Mengerian and thus the following holds.

Lemma $6.1 \ell$ is neither a loop nor a coloop of $M$.

The next lemma can be proved easily from the definition of TDI systems, so we omit its proof.

Lemma 6.2 Suppose the system $C_{i} \boldsymbol{x}_{i} \geq \boldsymbol{d}_{i}, \boldsymbol{x}_{i} \geq \mathbf{0}$ is TDI, for $i=1,2$. Then so is the system

$$
\left[\begin{array}{cc}
C_{1} & 0 \\
0 & C_{2}
\end{array}\right]\left[\begin{array}{l}
\boldsymbol{x}_{1} \\
\boldsymbol{x}_{2}
\end{array}\right] \geq\left[\begin{array}{l}
\boldsymbol{d}_{1} \\
\boldsymbol{d}_{2}
\end{array}\right], \quad\left[\begin{array}{l}
\boldsymbol{x}_{1} \\
\boldsymbol{x}_{2}
\end{array}\right] \geq\left[\begin{array}{l}
\mathbf{0} \\
\mathbf{0}
\end{array}\right]
$$

A straightforward consequence of this lemma is the following observation. Suppose the system $C \boldsymbol{x} \geq \boldsymbol{d}$, $\boldsymbol{x} \geq \mathbf{0}$ is box-TDI. If $C^{\prime}$ is obtained from $C$ by adding a zero-column, then the system $C^{\prime} \boldsymbol{x}^{\prime} \geq \boldsymbol{d}, \boldsymbol{x}^{\prime} \geq \mathbf{0}$ is also box-TDI. This observation in turn implies the following.

Lemma 6.3 $M$ is connected.

Putting Lemma 6.1 and Lemma 6.3 together we get the following.

Lemma $6.4(M, \ell) \in \Im$.

From this point on, it would be more convenient for us to view $\mathcal{P}_{M, \ell}$ as $\mathcal{O}_{N, \Sigma}$, as guaranteed by Proposition 3.1. In particular, since $A=A_{\mathcal{O}_{N, \Sigma}}$, we deduce the following from Lemma 6.2 immediately.

Lemma 6.5 $N$ is connected.

The last two lemmas guarantee that all results from the last section can be applied to $(M, \ell)$. Since $\mathcal{O}_{N, \Sigma}$ is not box-Mengerian, there exist rational vectors $\boldsymbol{l}$ and $\boldsymbol{u}$ and an integral vector $\boldsymbol{w}$ for which $\operatorname{Max}(A, \boldsymbol{l}, \boldsymbol{u}, \boldsymbol{w})$ is finite yet has no integral optimal solution. Next, we analyze optimal solutions of this problem.

Lemma 6.6 If $(\boldsymbol{\alpha}, \boldsymbol{\beta}, \boldsymbol{\gamma})$ is an optimal solution to $\operatorname{Max}(A, \boldsymbol{l}, \boldsymbol{u}, \boldsymbol{w})$ such that the flow $(\boldsymbol{f}, \boldsymbol{\alpha})$ is cross-free at a 2-separation $\left(E_{1}, E_{2}\right)$ of $N$, then $\boldsymbol{\alpha}\left(\mathcal{O}\left(E_{1}, E_{2}\right)\right)$ is not integral. 
Proof. Since $(\boldsymbol{f}, \boldsymbol{\alpha})$ is cross-free at $\left(E_{1}, E_{2}\right)$, we may assume, by symmetry, that $C \cap E_{1}$ is $\Sigma$-odd for all $C \in \mathcal{O}\left(E_{1}, E_{2}\right) \cap \operatorname{spt}(\boldsymbol{\alpha})$. Let $\Sigma_{1}=\Sigma \cap E_{1}$ and $\Sigma_{2}=\left(\Sigma \cap E_{2}\right) \cup\{p\}$. Let $N_{1}, N_{2}$ be the two matroids induced by $\left(E_{1}, E_{2}\right)$. By Lemma 3.2, both $\mathcal{O}_{N_{1}, \Sigma_{1}}$ and $\mathcal{O}_{N_{2}, \Sigma_{2}}$ are proper minors of $\mathcal{O}_{N, \Sigma}$ and thus, both are box-Mengerian, as $\mathcal{O}_{N, \Sigma}$ is a minor minimal counterexample.

Suppose $\boldsymbol{\alpha}\left(\mathcal{O}\left(E_{1}, E_{2}\right)\right)$ is integral. For $i=1,2$, let $A_{i}=A_{\mathcal{O}_{N_{i}, \Sigma_{i}}} ;$ let $\boldsymbol{\beta}_{i}, \boldsymbol{\gamma}_{i} \in \mathbb{Q}_{+}^{E_{i} \cup\{p\}}, \boldsymbol{l}_{i}, \boldsymbol{u}_{i} \in \mathbb{Q}^{E_{i} \cup\{p\}}$, and $\boldsymbol{w}_{i} \in \mathbb{Z}^{E_{i} \cup\{p\}}$ be such that $\left.\boldsymbol{\beta}_{i}\right|_{E_{i}}=\left.\boldsymbol{\beta}\right|_{E_{i}},\left.\boldsymbol{\gamma}_{i}\right|_{E_{i}}=\left.\gamma\right|_{E_{i}},\left.\boldsymbol{l}_{i}\right|_{E_{i}}=\left.\boldsymbol{l}\right|_{E_{i}},\left.\boldsymbol{u}_{i}\right|_{E_{i}}=\left.\boldsymbol{u}\right|_{E_{i}},\left.\boldsymbol{w}_{i}\right|_{E_{i}}=\left.\boldsymbol{w}\right|_{E_{i}}$, $\boldsymbol{\beta}_{i}(p)=\gamma_{i}(p)=\boldsymbol{l}_{i}(p)=0, \boldsymbol{u}_{i}(p)=+\infty$, and $\boldsymbol{w}_{i}(p)=\boldsymbol{\alpha}\left(\mathcal{O}\left(E_{1}, E_{2}\right)\right)$. Let $\left(\boldsymbol{f}_{1}, \boldsymbol{\alpha}_{1}\right)$ and $\left(\boldsymbol{f}_{2}, \boldsymbol{\alpha}_{2}\right)$ be the components of $(\boldsymbol{f}, \boldsymbol{\alpha})$. Then, for $i=1,2,\left(\boldsymbol{\alpha}_{i}, \boldsymbol{\beta}_{i}, \boldsymbol{\gamma}_{i}\right)$ is a feasible solution to $\operatorname{Max}\left(A_{i}, \boldsymbol{l}_{i}, \boldsymbol{u}_{i}, \boldsymbol{w}_{i}\right)$.

We claim that, for $i=1,2 \operatorname{Max}\left(A_{i}, \boldsymbol{l}_{i}, \boldsymbol{u}_{i}, \boldsymbol{w}_{i}\right)$ has a finite maximum. To see this, we consider any of its feasible solution $\left(\boldsymbol{\alpha}_{i}^{\prime}, \boldsymbol{\beta}_{i}^{\prime}, \boldsymbol{\gamma}_{i}^{\prime}\right)$. Let $\left(\boldsymbol{f}^{\prime}, \boldsymbol{\alpha}^{\prime}\right)$ be the sum of $\left(\boldsymbol{f}_{1}^{\prime}, \boldsymbol{\alpha}_{1}^{\prime}\right)$ and $\left(\boldsymbol{f}_{2}^{\prime}, \boldsymbol{\alpha}_{2}^{\prime}\right)$. Let $\boldsymbol{\beta}^{\prime}, \boldsymbol{\gamma}^{\prime} \in \mathbb{Q}_{+}^{E}$ be such that $\left.\boldsymbol{\beta}^{\prime}\right|_{E_{i}}=\left.\boldsymbol{\beta}_{i}^{\prime}\right|_{E_{i}}$ and $\left.\boldsymbol{\gamma}^{\prime}\right|_{E_{i}}=\left.\boldsymbol{\gamma}_{i}^{\prime}\right|_{E_{i}}$, for $i=1,2$. Then Lemma 5.3 implies that $\left(\boldsymbol{\alpha}^{\prime}, \boldsymbol{\beta}^{\prime}, \boldsymbol{\gamma}^{\prime}\right)$ is a feasible solution to $\operatorname{Max}(A, \boldsymbol{l}, \boldsymbol{u}, \boldsymbol{w})$. Moreover,

$$
\begin{aligned}
& \left(\boldsymbol{\alpha}_{1}^{\prime}\right)^{T} \mathbf{1}+\left(\boldsymbol{\beta}_{1}^{\prime}\right)^{T} \boldsymbol{l}_{1}-\left(\boldsymbol{\gamma}_{1}^{\prime}\right)^{T} \boldsymbol{u}_{1}+\left(\boldsymbol{\alpha}_{2}^{\prime}\right)^{T} \mathbf{1}+\left(\boldsymbol{\beta}_{2}^{\prime}\right)^{T} \boldsymbol{l}_{2}-\left(\boldsymbol{\gamma}_{2}^{\prime}\right)^{T} \boldsymbol{u}_{2} \\
\leq & \left(\boldsymbol{\alpha}_{1}^{\prime}\right)^{T} \mathbf{1}+\left(\boldsymbol{\alpha}_{2}^{\prime}\right)^{T} \mathbf{1}+\left(\boldsymbol{\beta}_{1}^{\prime}\right)^{T} \boldsymbol{l}_{1}+\left(\boldsymbol{\beta}_{2}^{\prime}\right)^{T} \boldsymbol{l}_{2}-\left(\boldsymbol{\gamma}^{\prime}\right)^{T} \boldsymbol{u} \\
= & \left(\boldsymbol{\alpha}^{\prime}\right)^{T} \mathbf{1}+\left(\boldsymbol{\beta}^{\prime}\right)^{T} \boldsymbol{l}-\left(\boldsymbol{\gamma}^{\prime}\right)^{T} \boldsymbol{u}+\max \left\{\boldsymbol{f}_{1}^{\prime}(p), \boldsymbol{f}_{2}^{\prime}(p)\right\} \\
\leq & \boldsymbol{\alpha}^{T} \mathbf{1}+\boldsymbol{\beta}^{T} \boldsymbol{l}-\boldsymbol{\gamma}^{T} \boldsymbol{u}+\boldsymbol{\alpha}\left(\mathcal{O}\left(E_{1}, E_{2}\right)\right),
\end{aligned}
$$

where the last inequality holds since, for $i=1,2$, by the constraint of $\operatorname{Max}\left(A_{i}, \boldsymbol{l}_{i}, \boldsymbol{u}_{i}, \boldsymbol{w}_{i}\right)$ (recall (4.1)) we have $\boldsymbol{f}_{i}^{\prime}(p)+\boldsymbol{\beta}_{i}(p)-\boldsymbol{\gamma}_{i}(p) \leq \boldsymbol{w}_{i}(p)$. So $\boldsymbol{f}_{i}^{\prime}(p) \leq \boldsymbol{w}_{i}(p)=\boldsymbol{\alpha}\left(\mathcal{O}\left(E_{1}, E_{2}\right)\right)$. Our claim is thus justified.

For $i=1,2, \operatorname{Max}\left(A_{i}, \boldsymbol{l}_{i}, \boldsymbol{u}_{i}, \boldsymbol{w}_{i}\right)$ has an integral optimal solution $\left(\boldsymbol{\alpha}_{i}^{*}, \boldsymbol{\beta}_{i}^{*}, \boldsymbol{\gamma}_{i}^{*}\right)$, as $\mathcal{O}_{N_{i}, \Sigma_{i}}$ is box-Mengerian. Since $\boldsymbol{u}_{1}(p)=\boldsymbol{u}_{2}(p)=+\infty$, it follows that $\boldsymbol{\gamma}_{1}^{*}(p)=\boldsymbol{\gamma}_{2}^{*}(p)=0$. Let $\left(\boldsymbol{f}^{*}, \boldsymbol{\alpha}^{*}\right)$ be sum of $\left(\boldsymbol{f}_{1}^{*}, \boldsymbol{\alpha}_{1}^{*}\right)$ and $\left(\boldsymbol{f}_{2}^{*}, \boldsymbol{\alpha}_{2}^{*}\right)$. By Lemma 5.3, $\boldsymbol{\alpha}^{*}$ is integral. Let $\boldsymbol{\beta}^{*}, \boldsymbol{\gamma}^{*} \in \mathbb{Z}_{+}^{E}$ be such that $\left.\boldsymbol{\beta}^{*}\right|_{E_{i}}=\left.\boldsymbol{\beta}_{i}^{*}\right|_{E_{i}}$ and $\left.\boldsymbol{\gamma}^{*}\right|_{E_{i}}=\left.\boldsymbol{\gamma}_{i}^{*}\right|_{E_{i}}$, for $i=1,2$. Then $\left(\boldsymbol{\alpha}^{*}, \boldsymbol{\beta}^{*}, \boldsymbol{\gamma}^{*}\right)$ is a feasible solution to $\operatorname{Max}(A, \boldsymbol{l}, \boldsymbol{u}, \boldsymbol{w})$. Moreover,

$$
\begin{aligned}
\left(\boldsymbol{\alpha}^{*}\right)^{T} \mathbf{1}+\left(\boldsymbol{\beta}^{*}\right)^{T} \boldsymbol{l}-\left(\boldsymbol{\gamma}^{*}\right)^{T} \boldsymbol{u} & \geq\left(\boldsymbol{\alpha}_{1}^{*}\right)^{T} \mathbf{1}+\left(\boldsymbol{\alpha}_{2}^{*}\right)^{T} \mathbf{1}-\boldsymbol{w}_{1}(p)+\left(\boldsymbol{\beta}^{*}\right)^{T} \boldsymbol{l}-\left(\boldsymbol{\gamma}^{*}\right)^{T} \boldsymbol{u} \\
& =\left(\boldsymbol{\alpha}_{1}^{*}\right)^{T} \mathbf{1}+\left(\boldsymbol{\alpha}_{2}^{*}\right)^{T} \mathbf{1}-\boldsymbol{w}_{1}(p)+\left(\boldsymbol{\beta}_{1}^{*}\right)^{T} \boldsymbol{l}_{1}+\left(\boldsymbol{\beta}_{2}^{*}\right)^{T} \boldsymbol{l}_{2}-\left(\gamma_{1}^{*}\right)^{T} \boldsymbol{u}_{1}-\left(\gamma_{2}^{*}\right)^{T} \boldsymbol{u}_{2} \\
& \geq \boldsymbol{\alpha}_{1}^{T} \mathbf{1}+\boldsymbol{\beta}_{1}^{T} \boldsymbol{l}_{1}-\boldsymbol{\gamma}_{1}^{T} \boldsymbol{u}_{1}+\boldsymbol{\alpha}_{2}^{T} \mathbf{1}+\boldsymbol{\beta}_{2}^{T} \boldsymbol{l}_{2}-\boldsymbol{\gamma}_{2}^{T} \boldsymbol{u}_{2}-\boldsymbol{w}_{1}(p) \\
& =\boldsymbol{\alpha}^{T} \mathbf{1}+\boldsymbol{\beta}_{1}^{T} \boldsymbol{l}_{1}-\boldsymbol{\gamma}_{1}^{T} \boldsymbol{u}_{1}+\boldsymbol{\beta}_{2}^{T} \boldsymbol{l}_{2}-\boldsymbol{\gamma}_{2}^{T} \boldsymbol{u}_{2} \\
& =\boldsymbol{\alpha}^{T} \mathbf{1}+\boldsymbol{\beta}^{T} \boldsymbol{l}-\boldsymbol{\gamma}^{T} \boldsymbol{u}
\end{aligned}
$$

which implies that $\left(\boldsymbol{\alpha}^{*}, \boldsymbol{\beta}^{*}, \boldsymbol{\gamma}^{*}\right)$ is an integral optimal solution, a contradiction.

In general, there are many ways to choose $\boldsymbol{l}, \boldsymbol{u}$, and $\boldsymbol{w}$ so that $\operatorname{Max}(A, \boldsymbol{l}, \boldsymbol{u}, \boldsymbol{w})$ is finite yet has no integral optimal solutions. Let us call such a triple $(\boldsymbol{l}, \boldsymbol{u}, \boldsymbol{w})$ a certificate.

Lemma 6.7 There exists a certificate $(\boldsymbol{l}, \boldsymbol{u}, \boldsymbol{w})$ with $\boldsymbol{l} \geq \mathbf{0}$.

Proof. Let $(\boldsymbol{l}, \boldsymbol{u}, \boldsymbol{w})$ be any certificate and let $E^{-}=\{e \in E: \boldsymbol{l}(e)<0\}$. For any $\boldsymbol{z} \in \mathbb{Q}^{E}$, let $\tilde{\boldsymbol{z}} \in \mathbb{Q}^{E}$ denote the vector with $\tilde{\boldsymbol{z}}(e)=\max \{\boldsymbol{z}(e), 0\}$, for all $e \in E$. We show that $(\tilde{\boldsymbol{l}}, \boldsymbol{u}, \boldsymbol{w})$ is also a certificate, which proves the lemma. Since $\operatorname{Max}(A, \tilde{\boldsymbol{l}}, \boldsymbol{u}, \boldsymbol{w})$ and $\operatorname{Max}(A, \boldsymbol{l}, \boldsymbol{u}, \boldsymbol{w})$ have the same set of feasible solutions and 
$\tilde{\boldsymbol{l}} \geq \boldsymbol{l}$, so the value of $\operatorname{Max}(A, \tilde{\boldsymbol{l}}, \boldsymbol{u}, \boldsymbol{w})$ is at least that of $\operatorname{Max}(A, \boldsymbol{l}, \boldsymbol{u}, \boldsymbol{w})$. On the other hand, for any feasible solution $(\boldsymbol{\alpha}, \boldsymbol{\beta}, \boldsymbol{\gamma})$ of $\operatorname{Max}(A, \tilde{\boldsymbol{l}}, \boldsymbol{u}, \boldsymbol{w})$, let $\boldsymbol{\beta}^{\prime}$ be obtained from $\boldsymbol{\beta}$ by setting $\boldsymbol{\beta}^{\prime}(e)=0$ if $\boldsymbol{l}(e)<0$ and setting $\boldsymbol{\beta}^{\prime}(e)=\boldsymbol{\beta}(e)$ otherwise, for all $e \in E$. Then $\left(\boldsymbol{\alpha}, \boldsymbol{\beta}^{\prime}, \boldsymbol{\gamma}\right)$ is a feasible solution to $\operatorname{Max}(A, \boldsymbol{l}, \boldsymbol{u}, \boldsymbol{w})$ and $\boldsymbol{\beta}^{T} \tilde{\boldsymbol{l}}=$ $\left(\boldsymbol{\beta}^{\prime}\right)^{T} \boldsymbol{l}$, which implies that $\operatorname{Max}(A, \tilde{\boldsymbol{l}}, \boldsymbol{u}, \boldsymbol{w})$ and $\operatorname{Max}(A, \boldsymbol{l}, \boldsymbol{u}, \boldsymbol{w})$ have the same maximum. Consequently, $\operatorname{Max}(A, \tilde{\boldsymbol{l}}, \boldsymbol{u}, \boldsymbol{w})$ is finite. Notice that $\boldsymbol{\beta}^{\prime}$ is integral if $\boldsymbol{\beta}$ is. Therefore, if $(\boldsymbol{\alpha}, \boldsymbol{\beta}, \boldsymbol{\gamma})$ is an integral optimal solution of $\operatorname{Max}(A, \tilde{\boldsymbol{l}}, \boldsymbol{u}, \boldsymbol{w})$ then $\left(\boldsymbol{\alpha}, \boldsymbol{\beta}^{\prime}, \boldsymbol{\gamma}\right)$ would be an integral optimal solution of $\operatorname{Max}(A, \boldsymbol{l}, \boldsymbol{u}, \boldsymbol{w})$, which is impossible. Thus $(\tilde{\boldsymbol{l}}, \boldsymbol{u}, \boldsymbol{w})$ is a certificate, as required.

Lemma 6.8 Suppose $\boldsymbol{l} \geq \mathbf{0}$ and $\left(E_{1}, E_{2}\right)$ is a 2-separation of $N$. Let $(\boldsymbol{\alpha}, \boldsymbol{\beta}, \boldsymbol{\gamma})$ be chosen among all optimal solutions of $\operatorname{Max}(A, \boldsymbol{l}, \boldsymbol{u}, \boldsymbol{w})$ such that $\boldsymbol{\alpha}\left(\mathcal{O}\left(E_{1}, E_{2}\right)\right)$ is minimized. If the flow $(\boldsymbol{f}, \boldsymbol{\alpha})$ is connected and $\left(E_{1}, E_{2}\right)$-minimal then $\boldsymbol{\alpha}\left(\mathcal{O}\left(E_{1}, E_{2}\right)\right)$ is integral.

Proof. Suppose, on the contrary, that $\boldsymbol{\alpha}\left(\mathcal{O}\left(E_{1}, E_{2}\right)\right) \notin \mathbb{Z}$. Let $N_{1}$ and $N_{2}$ be the two matroids induced by $\left(E_{1}, E_{2}\right)$. Notice that $(\boldsymbol{f}, \boldsymbol{\alpha})$ is tight at $\left(E_{1}, E_{2}\right)$, with respect to $(\boldsymbol{f}, \boldsymbol{\alpha})$. By Lemma $5.1,(\boldsymbol{f}, \boldsymbol{\alpha})$ is cross-free at $\left(E_{1}, E_{2}\right)$. Thus we may consider the components $\left(\boldsymbol{f}_{1}, \boldsymbol{\alpha}_{1}\right)$ and $\left(\boldsymbol{f}_{2}, \boldsymbol{\alpha}_{2}\right)$ of $(\boldsymbol{f}, \boldsymbol{\alpha})$. By Lemma 5.4 and Lemma 5.2, for $i=1,2,\left(\boldsymbol{f}_{i}, \boldsymbol{\alpha}_{i}\right)$ is minimal and connected, and $\boldsymbol{f}_{i}(p)=\boldsymbol{\alpha}\left(\mathcal{O}\left(E_{1}, E_{2}\right)\right) \notin \mathbb{Z}$. Consequently, by Lemma 5.6, $N_{i}$ has an $\boldsymbol{f}_{i}$-fractional circuit $C_{i} \ni p$. Let $C=\left(C_{1}-\{p\}\right) \cup\left(C_{2}-\{p\}\right)$. Then $C \in \mathcal{O}\left(E_{1}, E_{2}\right)$ is $\boldsymbol{f}$-fractional. Let us apply Lemma 5.5 to $(\boldsymbol{f}, \boldsymbol{\alpha})$ and $C$. Let $t, \delta, \boldsymbol{\chi}, \varepsilon$, and $\left(\boldsymbol{f}_{ \pm}, \boldsymbol{\alpha}_{ \pm}\right)$satisfy the conclusion of the lemma. Let $\boldsymbol{\mu}, \boldsymbol{\rho} \in\{0, \pm 1\}^{E}$ be such that

$$
\boldsymbol{\mu}=\left\{\begin{array}{ll}
\chi(e) & \text { if } e \in C \text { and } \boldsymbol{f}(e)<\boldsymbol{w}(e) \\
0 & \text { otherwise }
\end{array} \text { and } \boldsymbol{\rho}= \begin{cases}\chi(e) & \text { if } e \in C \text { and } \boldsymbol{f}(e)>\boldsymbol{w}(e) \\
0 & \text { otherwise }\end{cases}\right.
$$

Since $\boldsymbol{w}$ is integral, it follows that $\boldsymbol{f}(e) \neq \boldsymbol{w}(e)$, for all $e \in C$, and thus $\boldsymbol{\mu}+\boldsymbol{\rho}=\boldsymbol{\chi}$.

Observe that $(\boldsymbol{\alpha}, \boldsymbol{w}-\boldsymbol{f}+\boldsymbol{\gamma}, \boldsymbol{\gamma})$ is also an optimal solution of $\operatorname{Max}(A, \boldsymbol{l}, \boldsymbol{u}, \boldsymbol{w})$, as $\boldsymbol{l} \geq \mathbf{0}$. Thus we may assume, without loss of generality, that $\boldsymbol{\beta}=\boldsymbol{w}-\boldsymbol{f}+\boldsymbol{\gamma}$. Let $\boldsymbol{\beta}_{ \pm}=\boldsymbol{\beta} \mp \varepsilon \boldsymbol{\mu}$ and $\boldsymbol{\gamma}_{ \pm}=\boldsymbol{\gamma} \pm \varepsilon \boldsymbol{\rho}$. Notice that $\boldsymbol{f}(e)<\boldsymbol{w}(e)$ implies $\boldsymbol{\beta}(e)>0$, and $\boldsymbol{f}(e)>\boldsymbol{w}(e)$ implies $\boldsymbol{\gamma}(e)>0$. Therefore, if $\varepsilon$ is sufficiently small, we have $\boldsymbol{\beta}_{ \pm} \geq \mathbf{0}$ and $\boldsymbol{\gamma}_{ \pm} \geq \mathbf{0}$. Furthermore,

$$
\boldsymbol{\beta}_{ \pm}-\boldsymbol{\gamma}_{ \pm}=\boldsymbol{\beta}-\boldsymbol{\gamma} \mp \varepsilon(\boldsymbol{\mu}+\boldsymbol{\rho})=\boldsymbol{\beta}-\boldsymbol{\gamma} \mp \varepsilon \boldsymbol{\chi}
$$

and so

$$
\boldsymbol{f}_{ \pm}+\boldsymbol{\beta}_{ \pm}-\gamma_{ \pm} \leq \boldsymbol{f} \pm \varepsilon \boldsymbol{\chi}+\boldsymbol{\beta}-\boldsymbol{\gamma} \mp \varepsilon \boldsymbol{\chi}=\boldsymbol{f}+\boldsymbol{\beta}-\boldsymbol{\gamma} \leq \boldsymbol{w},
$$

which implies that both $\left(\boldsymbol{\alpha}_{+}, \boldsymbol{\beta}_{+}, \boldsymbol{\gamma}_{+}\right)$and $\left(\boldsymbol{\alpha}_{-}, \boldsymbol{\beta}_{-}, \boldsymbol{\gamma}_{-}\right)$are feasible solutions to $\operatorname{Max}(A, \boldsymbol{l}, \boldsymbol{u}, \boldsymbol{w})$. Since

$$
\begin{aligned}
\boldsymbol{\alpha}^{T} \mathbf{1}+\boldsymbol{\beta}^{T} \boldsymbol{l}-\boldsymbol{\gamma}^{T} \boldsymbol{u} & \geq \boldsymbol{\alpha}_{ \pm}^{T} \mathbf{1}+\boldsymbol{\beta}_{ \pm}^{T} \boldsymbol{l}-\gamma_{ \pm}^{T} \boldsymbol{u} \\
& \geq \boldsymbol{\alpha}^{T} \mathbf{1} \pm t \varepsilon+\boldsymbol{\beta}^{T} \boldsymbol{l} \mp \varepsilon \boldsymbol{\mu}^{T} \mathbf{1}-\boldsymbol{\gamma}^{T} \boldsymbol{u} \mp \varepsilon \boldsymbol{\rho}^{T} \boldsymbol{u} \\
& =\boldsymbol{\alpha}^{T} \mathbf{1}+\boldsymbol{\beta}^{T} \boldsymbol{l}-\boldsymbol{\gamma}^{T} \boldsymbol{u} \pm \varepsilon\left(t-\boldsymbol{\mu}^{T} \mathbf{1}-\boldsymbol{\rho}^{T} \boldsymbol{u}\right),
\end{aligned}
$$

so we must have $t-\boldsymbol{\mu}^{T} \mathbf{1}-\boldsymbol{\rho}^{T} \boldsymbol{u}=0$, and thus both $\left(\boldsymbol{\alpha}_{+}, \boldsymbol{\beta}_{+}, \boldsymbol{\gamma}_{+}\right)$and $\left(\boldsymbol{\alpha}_{-}, \boldsymbol{\beta}_{-}, \boldsymbol{\gamma}_{-}\right)$are optimal solutions. However, by Lemma 5.5, $\min \left\{\boldsymbol{\alpha}_{ \pm}\left(\mathcal{O}\left(E_{1}, E_{2}\right)\right)\right\} \leq \boldsymbol{\alpha}\left(\mathcal{O}\left(E_{1}, E_{2}\right)\right)-\varepsilon$, which contradicts the minimality of $\boldsymbol{\alpha}\left(\mathcal{O}\left(E_{1}, E_{2}\right)\right)$, and thus the lemma is proved. 
Now we are ready to prove our main result.

Proof of Theorem 1.3. The necessity was proved in Section 2, so we only prove the sufficiency. Suppose the result is false. Then there is a minor minimal counterexample $(M, \ell)$. By Lemma $6.4,(M, \ell) \in \Im$, and by Lemma $6.5, N=M / \ell$ is connected. By Lemma $4.3, M$ is not regular, which implies, by Theorem 3.4, that $N$ has a 2-separation $\left(E_{1}, E_{2}\right)$. Let $\Sigma \subseteq E$ such that $\Sigma \cup\{\ell\}$ is a cocycle of $M$. Then Proposition 3.1 implies that $\mathcal{P}_{M, \ell}=\mathcal{O}_{N, \Sigma}$. Let $A$ be the $\mathcal{P}_{M, \ell^{-}} E$ incidence matrix. Let $\boldsymbol{l}, \boldsymbol{u} \in \mathbb{Q}^{E}$ and $\boldsymbol{w} \in \mathbb{Z}^{E}$ such that $\operatorname{Max}(A, \boldsymbol{l}, \boldsymbol{u}, \boldsymbol{w})$ is finite yet has no integral optimal solution.

Claim 1. For any optimal solution $(\boldsymbol{\alpha}, \boldsymbol{\beta}, \boldsymbol{\gamma})$ of $\operatorname{Max}(A, \boldsymbol{l}, \boldsymbol{u}, \boldsymbol{w})$, the flow $(\boldsymbol{f}, \boldsymbol{\alpha})$ is connected.

Suppose otherwise. Then there exists a flow $\left(\boldsymbol{f}^{\prime}, \boldsymbol{\alpha}^{\prime}\right) \preceq(\boldsymbol{f}, \boldsymbol{\alpha})$ such that $\boldsymbol{\alpha}^{\prime}\left(\mathcal{O}\left(E_{1}, E_{2}\right)\right)=0$, for some 2-separation $\left(E_{1}, E_{2}\right)$ of $N$. From $\left(\boldsymbol{f}^{\prime}, \boldsymbol{\alpha}^{\prime}\right) \preceq(\boldsymbol{f}, \boldsymbol{\alpha})$ we deduce that $\left(\boldsymbol{\alpha}^{\prime}, \boldsymbol{\beta}, \boldsymbol{\gamma}\right)$ is also an optimal solution of $\operatorname{Max}(A, \boldsymbol{l}, \boldsymbol{u}, \boldsymbol{w})$; from $\boldsymbol{\alpha}^{\prime}\left(\mathcal{O}\left(E_{1}, E_{2}\right)\right)=0$ we deduce that $\left(\boldsymbol{f}^{\prime}, \boldsymbol{\alpha}^{\prime}\right)$ is tight, and hence by Lemma 5.1, $\left(\boldsymbol{f}^{\prime}, \boldsymbol{\alpha}^{\prime}\right)$ is cross-free at $\left(E_{1}, E_{2}\right)$. Then Lemma 6.6 implies that $\boldsymbol{\alpha}^{\prime}\left(\mathcal{O}\left(E_{1}, E_{2}\right)\right)$ is not integral, which is a contradiction, so the claim is proved.

By Lemma 6.7, we may assume that $\boldsymbol{l} \geq \mathbf{0}$. Let us choose an optimal solution $(\boldsymbol{\alpha}, \boldsymbol{\beta}, \boldsymbol{\gamma})$ such that

(i) $\boldsymbol{\alpha}\left(\mathcal{O}\left(E_{1}, E_{2}\right)\right)$ is minimized;

(ii) subject to (i), $\boldsymbol{f}(E)$ is minimized, where $\boldsymbol{f}=A^{T} \boldsymbol{\alpha}$.

Claim 2. $\boldsymbol{\alpha}\left(\mathcal{O}\left(E_{1}, E_{2}\right)\right)$ is not integral.

Our choice (i) implies that $(\boldsymbol{f}, \boldsymbol{\alpha})$ is tight at $\left(E_{1}, E_{2}\right)$, with respect to itself. By Lemma $5.1,(\boldsymbol{f}, \boldsymbol{\alpha})$ is cross-free, and thus the claim follows from Lemma 6.6.

Claim 3. The flow $(\boldsymbol{f}, \boldsymbol{\alpha})$ is $\left(E_{1}, E_{2}\right)$-minimal.

Consider any flow $\left(\boldsymbol{f}^{\prime}, \boldsymbol{\alpha}^{\prime}\right) \preceq(\boldsymbol{f}, \boldsymbol{\alpha})$ with $\boldsymbol{\alpha}^{\prime}\left(\mathcal{O}\left(E_{1}, E_{2}\right)\right) \leq \boldsymbol{\alpha}\left(\mathcal{O}\left(E_{1}, E_{2}\right)\right)$. Since $\left(\boldsymbol{\alpha}^{\prime}, \boldsymbol{\beta}, \boldsymbol{\gamma}\right)$ is feasible and $(\boldsymbol{\alpha}, \boldsymbol{\beta}, \boldsymbol{\gamma})$ is optimal, $\left(\boldsymbol{\alpha}^{\prime}, \boldsymbol{\beta}, \boldsymbol{\gamma}\right)$ must also be optimal and thus $\left(\boldsymbol{\alpha}^{\prime}\right)^{T} \mathbf{1}=\boldsymbol{\alpha}^{T} \mathbf{1}$ holds. By our choice (i), $\boldsymbol{\alpha}^{\prime}\left(\mathcal{O}\left(E_{1}, E_{2}\right)\right)=\boldsymbol{\alpha}\left(\mathcal{O}\left(E_{1}, E_{2}\right)\right)$. Thus our choice (ii) implies that $\boldsymbol{f}(E) \leq \boldsymbol{f}^{\prime}(E)$. However, our choice of $\boldsymbol{f}^{\prime}$ implies $\boldsymbol{f}^{\prime} \leq \boldsymbol{f}$, so we must have $\boldsymbol{f}^{\prime}=\boldsymbol{f}$, which proves the claim.

Now, by Claim 1, Claim 3, and Lemma 6.8, $\boldsymbol{\alpha}\left(\mathcal{O}\left(E_{1}, E_{2}\right)\right)$ is integral, which contradicts Claim 2. This contradiction proves that there is no counterexample and thus the theorem is proved.

\section{Applications}

In this section we discuss several applications of our main result.

\subsection{Box $\frac{1}{d}$-integral binary clutters}

Let $d$ be a positive integer. A linear system $A \boldsymbol{x} \geq \boldsymbol{b}$ is box $\frac{1}{d}$-integral if, for all $\frac{1}{d}$-integral vectors $\boldsymbol{u}$ and $\boldsymbol{l}$, all vertices of $\{\boldsymbol{x}: A \boldsymbol{x} \geq \boldsymbol{b}, \boldsymbol{u} \geq \boldsymbol{x} \geq \boldsymbol{l}\}$ are $\frac{1}{d}$-integral.

Lemma 7.1 If $A \boldsymbol{x} \geq \boldsymbol{b}$ is box-TDI and $\boldsymbol{b}$ is integral, then $A \boldsymbol{x} \geq \boldsymbol{b}$ is box $\frac{1}{d}$-integral, for every integer $d>0$. 
Proof. For any integer $d>0$, observe that $\frac{1}{d} A \boldsymbol{x} \geq \boldsymbol{b}$ is also box-TDI. Let $\boldsymbol{u}$ and $\boldsymbol{l}$ be $\frac{1}{d}$-integral. Then the system $\frac{1}{d} A \boldsymbol{x} \geq \boldsymbol{b}, d \boldsymbol{u} \geq \boldsymbol{x} \geq d \boldsymbol{l}$ is TDI, which implies that the polyhedron $\boldsymbol{P}=\left\{\boldsymbol{x}: \frac{1}{d} A \boldsymbol{x} \geq \boldsymbol{b}, d \boldsymbol{u} \geq \boldsymbol{x} \geq d \boldsymbol{l}\right\}$ is integral. Since $d\{\boldsymbol{x}: A \boldsymbol{x} \geq \boldsymbol{b}, \boldsymbol{u} \geq \boldsymbol{x} \geq \boldsymbol{l}\}=\boldsymbol{P}$, the result follows.

For any clutter $\mathcal{L}$ on $E$, let $A$ be the $\mathcal{L}$-E incidence matrix. We call $\mathcal{L}$ box $\frac{1}{d}$-integral, where $d$ is a positive integer, if the system $A \boldsymbol{x} \geq \mathbf{1}, \boldsymbol{x} \geq \mathbf{0}$ is box $\frac{1}{d}$-integral.

Theorem. (Gerards and Laurent [9]) The following are equivalent for every binary clutter $\mathcal{L}$.

(i) $\mathcal{L}$ has neither $Q_{6}$ nor $Q_{7}$ minor;

(ii) $\mathcal{L}$ is box $\frac{1}{d}$-integral for some integer $d \geq 2$;

(iii) $\mathcal{L}$ is box $\frac{1}{d}$-integral for all integers $d \geq 1$.

The implications $(i i i) \Rightarrow(i i) \Rightarrow(i)$ are given in [9]. The difficult part for proving this theorem, $(i) \Rightarrow(i i i)$, follows from Lemma 7.1 and Corollary 1.2.

\subsection{Odd cycles in signed graphs}

A signed graph is a graph $G$ together with a subset $\Sigma$ of $E(G)$. A cycle of $G$ is odd if it contains an odd number of edges from $\Sigma$. It is well known $[18,9]$ that the set of edge sets of odd cycles in a signed graph forms a binary clutter $\mathcal{C}_{G, \Sigma}$. More on signed graphs and odd cycles can be found in [4, 15].

Corollary 7.1 The following are equivalent for every signed graph $(G, \Sigma)$.

(i) $\mathcal{C}_{G, \Sigma}$ is Mengerian;

(ii) $\mathcal{C}_{G, \Sigma}$ is box-Mengerian;

(iii) $(G, \Sigma)$ cannot be reduced to $\left(K_{4}, E\left(K_{4}\right)\right)$ by the following operations:

(a) deleting an edge of $G$;

(b) contracting an edge not in $\Sigma$;

(c) replacing $\Sigma$ with the symmetric difference of $\Sigma$ and $\{x y \in E(G): x \in X, y \notin X\}$, for some $X \subseteq V(G)$.

It is explained in [9] that taking clutter minors in $\mathcal{C}_{G, \Sigma}$ is equivalent to reducing a signed graph using operations stated in (iii). Moreover, $Q_{6}$ equals $\mathcal{C}_{K_{4}, E\left(K_{4}\right)}$ yet $Q_{7}$ does not equal any $\mathcal{C}_{G, \Sigma}$. Therefore, the equivalence $(i) \Leftrightarrow($ iii) is a special case of Theorem 1.1 and the equivalence $(i i) \Leftrightarrow($ iii) follows from Corollary 1.2. We point out that this result strengthens Theorem 1.1 for the case of signed graphs.

\section{$7.3 \quad$ T-joins}

A graft [19] is a graph $G=(V, E)$ together with a subset $T \subseteq V$ of an even cardinality. The following figure shows three grafts, which we denote by $\mathcal{G}_{1}, \mathcal{G}_{2}$, and $\mathcal{G}_{3}$.
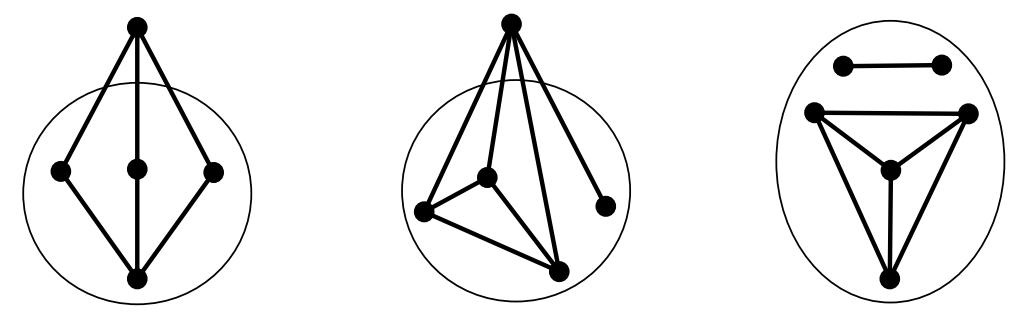
A subset $F \subseteq E$ is a $T$-join if $G \backslash(E-F)$ is a forest and its vertices of odd degree are precisely those in $T$. The set of all $T$-joins forms a binary clutter $[19,4,15]$. To see this, we define a binary matroid on $E \cup\{\ell\}$, where $\ell$ is an element not in $E$, by associating each element of $E \cup\{\ell\}$ with a vector over $G F(2)$. For each $v \in V$, let $\boldsymbol{a}_{v} \in\{0,1\}^{V}$ be the unit vector with $\boldsymbol{a}_{v}(v)=1$. Let $\boldsymbol{m}_{\ell}=\sum_{v \in T} \boldsymbol{a}_{v}$. For each $e=u v \in E$, let $\boldsymbol{m}_{e}=\boldsymbol{a}_{u}+\boldsymbol{a}_{v}$. Notice that, if $e$ is a loop then $\boldsymbol{m}_{e}=\mathbf{0}$, since the sum is taken over $G F(2)$. Let $M_{G, T}$ be the binary matroid on $E \cup\{\ell\}$ determined by vectors $\boldsymbol{m}_{e}(e \in E)$ and $\boldsymbol{m}_{\ell}$. As pointed out in [19], the $\ell$-port of $M_{G, T}$ is exactly the clutter of $T$-joins of $G$.

Taking minors in a $T$-join clutter can be translated into the following reductions in a graft [19]:

(i) deleting an edge;

(ii) contracting an edge $u v$, and putting the new vertex in $T$ if and only if $|T \cap\{u, v\}|=1$.

If Theorem 1.1 is restricted to this case, it says that $[4,15]$ the clutter of $T$-joins of a graph $G$ is Mengerian if and only if $(G, T)$ cannot be reduced to $\mathcal{G}_{1}$. The following is the restriction of Theorem 1.2 to this case.

Corollary 7.2 The clutter of T-joins of a graph $G$ is box-Mengerian if and only if none of $\mathcal{G}_{1}, \mathcal{G}_{2}$, and $\mathcal{G}_{3}$ can be reduced from $(G, T)$.

Proof. Observe that the $T$-join clutters of $\mathcal{G}_{1}, \mathcal{G}_{2}$, and $\mathcal{G}_{3}$ are $Q_{6}, Q_{7}$, and $Q_{7}$, respectively. In fact, it is well known $[4,15]$ that $\mathcal{G}_{1}$ is the only graft for which its $T$-join clutter is $Q_{6}$. By Corollary 1.2, we only need to verify that $\mathcal{G}_{2}$ and $\mathcal{G}_{3}$ are the only grafts for which their $T$-join clutters are $Q_{7}$.

Suppose the $T$-join clutter of $(G, T)$ is $Q_{7}$. Then $M_{G, T}$ (defined above) is $F_{7}^{+}$, with $\ell$ being a series element. It follows that $M_{G, T} \backslash \ell$ is the 1-sum of a coloop and $F_{7} \backslash e \cong M\left(K_{4}\right)$. In other words, $G$ has two blocks, $K_{2}$ and $K_{4}$. Depending on if $G$ is connected, there are two possibilities, which we denote by $G_{2}$ and $G_{3}$. Let $A_{G}$ be the vertex-edge incidence matrix of $G$. Then the graphic matroid $M(G)=M_{G, T} \backslash \ell$ is given by $A_{G}$, which implies that $M_{G, T}=F_{7}^{+}$can be expressed as a matrix $A_{G}^{*}$ obtained from $A_{G}$ by adding a column, corresponding $\ell$. Since $\ell$ is in series with the coloop of $G$, this new column is uniquely determined. It is routine to verify that the last column of $A_{G_{2}}^{*}$ has four 1's and the last column of $A_{G_{3}}^{*}$ has six 1's, which means that the corresponding grafts are $\mathcal{G}_{2}$ and $\mathcal{G}_{3}$.

Remark. If $T=\{s, t\}$ then $(G, T)$ cannot be reduced to any of $\mathcal{G}_{1}, \mathcal{G}_{2}$, and $\mathcal{G}_{3}$. Notice that, in this case, $T$-joins are $s t$-paths. So this corollary implies that the clutter of $s t$-paths of any graph is box-Mengerian.

\subsection{T-cuts}

Let $\mathcal{G}=(G, T)$ be a graft. A $T$-cut is a set of edges $\{x y \in E(G): x \in X, y \in Y\}$, for some partition $(X, Y)$ of $V(G)$ such that $|X \cap T|$ and $|Y \cap T|$ are odd. The set of $T$-cuts is a binary clutter. In fact, the $T$-cut clutter is the $\ell$-port of the dual of $M_{G, T}$, the binary matroid we defined in the last section. In the following result, the reduction we refer to are the reduction operations defined in the last section.

Corollary 7.3 The following are equivalent for every graft $(G, T)$.

(i) $(G, T)$ cannot be reduced to the graft $\left(K_{4}, V\left(K_{4}\right)\right)$;

(ii) the clutter of $T$-cuts of $G$ is Mengerian;

(iii) the clutter of $T$-cuts of $G$ is box-Mengerian.

Proof. The equivalence $(i) \Leftrightarrow(i i)$ follows $[4,15]$ from Theorem 1.1. To complete our proof, we only need to show that no $T$-cut clutter is $Q_{7}$. This is clear because if the $T$-cut clutter of a graph $G$ is $Q_{7}$, then 
$M_{G, T} \cong\left(F_{7}^{+}\right)^{*}$, which implies $F_{7}^{*}=\left(F_{7}^{+}\right)^{*} \backslash \ell \cong M_{G, T} \backslash \ell=M(G)$, the graphic matroid of $G$. This is a contradiction since $F_{7}^{*}$ is not graphic.

Remark. This corollary strengthens Theorem 1.1 when restricted to $T$-cuts. This case includes two special interesting cases, $|T|=2$ or $G$ is series-parallel.

\subsection{Two-commodity flows}

Let $s_{1}, s_{2}, t_{1}, t_{2}$ be vertices of a graph $G=(V, E)$ with $s_{1} \neq t_{1}$ and $s_{2} \neq t_{2}$. A two-commodity path is an $s_{1} t_{1^{-}}$or an $s_{2} t_{2}$-path. As pointed out in [18], two-commodity paths form a binary clutter. Moreover, it is routine to verify that, if $\mathcal{C}_{G}$ is the clutter of two-commodity paths of $G$, then for any $e \in E, \mathcal{C}_{G} \backslash e=\mathcal{C}_{G \backslash e}$ and $\mathcal{C}_{G} / e=\mathcal{C}_{G / e}$, except for $\mathcal{C}_{G} / e=\{\emptyset\}$, when $e=s_{i} t_{i}$, for some $i=1,2$. Therefore,

Proposition 7.1 If a clutter is the clutter of two-commodity paths of a graph, then so are all its minors.

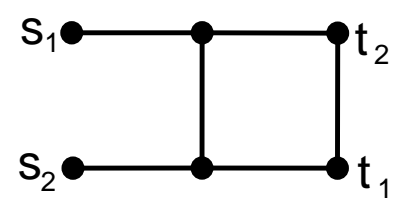

Corollary 7.4 The following are equivalent.

(i) the clutter of two-commodity paths of $G$ is Mengerian;

(ii) the clutter of two-commodity paths of $G$ is box-Mengerian;

(iii) no subgraph of $G$ can be contracted to the above graph, up to permuting indices and exchanging $s_{i}$ with $t_{i}$.

Proof. It has been pointed out in $[18,15]$ that the above graph is the only one for which $Q_{6}$ is its clutter of two-commodity paths. Therefore, the equivalence $(i) \Leftrightarrow($ iii $)$ follows form Proposition 7.1 and Theorem 1.1. To establish $(i i) \Leftrightarrow(i i i)$, by Proposition 7.1 and Corollary 1.2, we only need to show that $Q_{7}$ is not a two-commodity path clutter.

Suppose $G=(V, E)$ is a counterexample, where $E=\{1,2, \ldots, 7\}$. Without loss of generality, let $G$ be connected. Let $x$ and $y$ be the ends of edge 7 . Since 7 belongs to all members of $Q_{7}, G \backslash 7$ has two components $G_{x}$ and $G_{y}$, which contain $x$ and $y$, respectively. By symmetry, let us assume that 147 and 257 are $s_{1} t_{1}$-paths. Since $G$ is simple, edges $1,2,4,5$ must all belong to one of $G_{x}$ and $G_{y}$, say $G_{x}$. Notice that, in addition to edge $7, x$ is incident with one edge in $\{1,4\}$ and one edge in $\{2,5\}$. Consequently, at least one of the triples $127,157,427$, and 457 share a common vertex, $x$. However, this is impossible since each of these triples is a subset of a member of $Q_{7}$, which is supposed to be a set of paths of $G$.

Remark. This corollary strengthens Theorem 1.1 when restricted to two-commodity paths.

Let $G, s_{1}, t_{1}, s_{2}$, and $t_{2}$ be as before. A two-commodity cut is a minimal set of edges separating both $s_{1}$ from $t_{1}$, and $s_{2}$ from $t_{2}$. All two-commodity cuts of $G$ form a binary clutter $[18,4,15]$. In fact, as pointed out in [18], if $M$ is the matroid on $E \cup\{\ell\}$ such that its $\ell$-port is the two-commodity path clutter of $G$, then the $\ell$-port of $M^{*}$, the dual of $M$, is the two-commodity cut clutter of $G$, and vise versa.

Let $G_{0}$ be the first graph shown below. It is known $[18,15]$ that the two-commodity cut clutter of $G$ is Mengerian if and only if no subgraph of $G$ can be contracted to $G_{0}$, up to permuting indices and exchanging $s_{i}$ with $t_{i}$. The next is a characterization of box-Mengerian two-commodity cut clutters. 

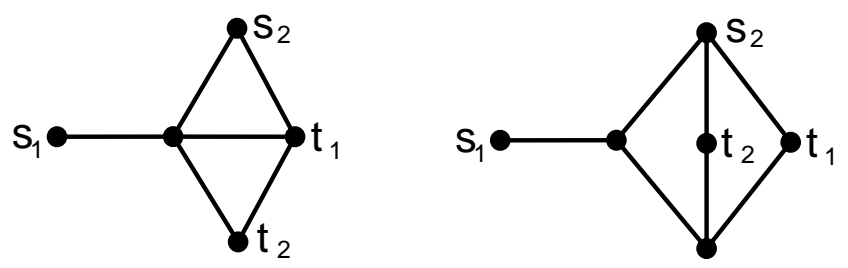

Corollary 7.5 The clutter of two-commodity cuts of $G$ is box-Mengerian if and only if no subgraph of $G$ can be contracted to any of the above two graphs, up to permuting indices and exchanging $s_{i}$ with $t_{i}$.

Proof. Let $G_{1}$ be the second graph shown above. Like before, we only need to show that $G_{1}$ is the only graph for which its two-commodity cut clutter is $Q_{7}$.

Suppose $G=(V, E)$ is such a graph. Then the two-commodity cut clutter of $G$ is the $\ell$-port of $F_{7}^{+}$. It follows $[16,18]$ that the the two-commodity path clutter of $G$ is the $\ell$-port of $\left(F_{7}^{+}\right)^{*}$. Notice that $\left(F_{7}^{+}\right)^{*}$ can also be obtained from $F_{7}^{*}$ by adding a parallel element $\ell$. Thus the two-commodity path clutter of $G$, which is the $\ell$-port of $\left(F_{7}^{+}\right)^{*}$, consists of a singleton $\{7\}$, and all members of $Q_{6}$, the $\ell$-port of $F_{7}^{*}$. Consequently, $G$ is obtained from the graph in Corollary 7.4 by adding an edge $s_{i} t_{i}$, for $i=1$ or 2 . Notice that the two resulting graphs, for $i=1,2$, are both isomorphic to $G_{1}$, so our result is proved.

\section{References}

[1] R.E. Bixby, $\ell$-matrices and a characterization of binary matroids, Discrete Mathematics 8 (1974) 139145.

[2] X. Chen, G. Ding, and W. Zang, The box-TDI system associated with 2-edge connected spanning subgraphs, submitted to Discrete Applied Mathematics.

[3] W. Cook, On box totally dual integral polyhedra, Mathematical Programming 34 (1986) 48-61.

[4] G. Cornuéjols, Combinatorial Optimization: Packing and Covering, SIAM, 2001.

[5] G. Ding and W. Zang, Packing circuits in matroids, Mathematical Programming Series A, to appear.

[6] J. Edmonds and D.R. Fulkerson, Bottleneck extrema, Journal of Combinatorial Theory 8 (1970) 299306.

[7] J. Edmonds and R. Giles, A min-max relation for submodular functions on graphs, Annals of Discrete Mathematics 1 (1977) 185-204.

[8] J. Edmonds and R. Giles, Total dual integrality of linear inequality systems, in: Progress in combinatorial optimization (W.R. Pulleyblank, ed.), Academic Press, Toronto (1984) 117-129.

[9] A.M.H. Gerards and M. Laurent, A characterization of box $\frac{1}{d}$-integral binary clutters, Journal of Combinatorial Theory Series B 65 (1995) 186-207.

[10] B. Guenin, A short proof of Seymour's characterization of the matroids with the max-flow min-cut property, Journal of Combinatorial Theory Series B 86 (2002) 273-279.

[11] M. Laurent and S. Poljak, One-third-integrality in the max-cut problem, Mathematical Programming 71 (1995) 29-50.

[12] A. Lehman, Matroids and ports, Notices of the American Mathematical Society 12 (1965) 342.

[13] J. Oxley, Matroid Theory, Oxford University Press, Oxford, 1992. 
[14] A. Schrijver, Theory of Linear and Integer Programming, John Wiley \& Sons, New York, 1986.

[15] A. Schrijver, Combinatorial Optimization - Polyhedra and Efficiency, Springer-Verlag, Berlin, 2003

[16] P.D. Seymour, The forbidden minors of binary clutters, Journal of the London Mathematical Society 12 (1976) 356-360.

[17] P.D. Seymour, A note on the production of matroid minors, Journal of Combinatorial Theory Series B 22 (1977) 289-295.

[18] P.D. Seymour, The matroids with the max-flow min-cut property, Journal of Combinatorial Theory Series B 23 (1977) 189-222.

[19] P.D. Seymour, Decomposition of regular matroids, Journal of Combinatorial Theory Series B 28 (1980) 305-359.

[20] K. Truemper, Matroid Decomposition, Academic Press, 1992.

[21] F.T. Tseng and K. Truemper, A decomposition of the matroids with the max-flow min-cut property, Discrete Applied Mathematics 15 (1986) 329-364.

[22] W.T. Tutte, A class of abelian groups, Canadian Journal of Mathematics 8 (1956) 13-28. 